\title{
ALMA observations of molecular tori around massive black holes ${ }^{\star}$
}

\author{
F. Combes ${ }^{1,2}$, S. García-Burillo ${ }^{3}$, A. Audibert ${ }^{1}$, L. Hunt ${ }^{4}$, A. Eckart ${ }^{5}$, S. Aalto ${ }^{6}$, V. Casasola ${ }^{4,7}$, F. Boone ${ }^{8}$, M. Krips ${ }^{9}$, \\ S. Viti ${ }^{10}$, K. Sakamoto ${ }^{11}$, S. Muller ${ }^{6}$, K. Dasyra ${ }^{12,13}$, P. van der Werf ${ }^{14}$, and S. Martin ${ }^{15,16}$
}

1 Observatoire de Paris, LERMA, CNRS, PSL Univ., Sorbonne University, UPMC, Paris, France

e-mail: francoise.combes@obspm.fr

2 College de France, 11 Pl. Marcelin Berthelot, 75231 Paris, France

3 Observatorio Astronómico Nacional (OAN)-Observatorio de Madrid, Alfonso XII, 3, 28014 Madrid, Spain

${ }^{4}$ INAF - Osservatorio Astrofisico di Arcetri, Largo E. Fermi, 5, 50125 Firenze, Italy

5 I. Physikalisches Institut, Universität zu Köln, Zülpicher Str. 77, 50937 Köln, Germany

${ }^{6}$ Dep. of Space, Earth and Environment, Chalmers University of Technology, Onsala Space Observatory, 43992 Onsala, Sweden

7 INAF - Istituto di Radioastronomia, Via Piero Gobetti 101, 40129 Bologna, Italy

8 CNRS, IRAP, 9 Av. colonel Roche, BP 44346, 31028 Toulouse Cedex 4, France

9 RAM, 300 rue de la Piscine, Domaine Universitaire, 38406 Saint Martin d'Hères, France

10 Dep. of Physics and Astronomy, UCL, Gower Place, London WC1E 6BT, UK

11 Academia Sinica, Institute of Astronomy and Astrophysics, Taiwan

12 Dep. of Astrophysics, Astronomy \& Mechanics, Faculty of Physics, National and Kapodistrian University of Athens, Panepistimiopolis, Zografou 15784, Greece

13 National Observatory of Athens, Institute for Astronomy, Astrophysics, Space Applications and Remote Sensing, Penteli 15236 Athens, Greece

${ }^{14}$ Leiden Observatory, Leiden Univ., PO Box 9513, 2300 RA Leiden, The Netherlands

15 European Southern Observatory, Alonso de Córdova, 3107, Vitacura, Santiago 763-0355, Chile

16 Joint ALMA Observatory, Alonso de Córdova, 3107 Vitacura, Santiago 763-0355, Chile

Received 2 November 2018 / Accepted 26 January 2019

\begin{abstract}
We report Atacama Large Millimeter/submillimeter Array (ALMA) observations of CO(3-2) emission in a sample of seven Seyfert/LINER galaxies at the unprecedented spatial resolution of $0{ }^{\prime} 1=4-9 \mathrm{pc}$. Our aim is to explore the close environment of active galactic nuclei (AGN), and the dynamical structures leading to their fueling, through the morphology and kinematics of the gas inside the sphere of influence of the black hole. The selected galaxies host low-luminosity AGN and have a wide range of activity types (Seyferts 1 to 2, LINERs), and barred or ringed morphologies. The observed maps reveal the existence of circumnuclear disk structures, defined by their morphology and decoupled kinematics, in most of the sample. We call these structures molecular tori, even though they often appear as disks without holes in the center. They have varying orientations along the line of sight, unaligned with the host galaxy orientation. The radius of the tori ranges from 6 to $27 \mathrm{pc}$, and their mass from $0.7 \times 10^{7}$ to $3.9 \times 10^{7} M_{\odot}$. The most edge-on orientations of the torus correspond to obscured Seyferts. In only one case (NGC 1365), the AGN is centered on the central gas hole of the torus. On a larger scale, the gas is always piled up in a few resonant rings $100 \mathrm{pc}$ in scale that play the role of a reservoir to fuel the nucleus. In some cases, a trailing spiral is observed inside the ring, providing evidence for feeding processes. More frequently, the torus and the AGN are slightly off-centered with respect to the bar-resonant ring position, implying that the black hole is wandering by a few $10 \mathrm{pc}$ amplitude around the center of mass of the galaxy. Our spatial resolution allows us to measure gas velocities inside the sphere of influence of the central black holes. By fitting the observations with different simulated cubes, varying the torus inclination and the black hole mass, it is possible to estimate the mass of the central black hole, which is in general difficult for such late-type galaxies, with only a pseudo-bulge. In some cases, AGN feedback is revealed through a molecular outflow, which will be studied in detail in a subsequent article.
\end{abstract}

Key words. galaxies: active - galaxies: ISM - galaxies: kinematics and dynamics - galaxies: nuclei - galaxies: spiral

\section{Introduction}

The growth of supermassive black holes in galaxies produces phenomenon of active galactic nuclei (AGN), one of the brightest and most energetic events in the Universe. In recent years, the subsequent appearance of AGN feedback has been widely established through the existence of fast outflows of ionized and atomic gas (e.g., Veilleux et al. 2005; Tombesi et al. 2010; Fiore et al. 2017). In parallel, observations of the molecular component of the circumnuclear environment have brought

\footnotetext{
* Based on observations carried out with ALMA in cycles 3 and 4.
}

a great deal of progress in the question of how AGN are fueled in galaxies (e.g., García-Burillo et al. 2005; Combes et al. 2013 , 2014), and how the energy generated by the AGN can in turn regulate its gas accretion through molecular outflows (e.g., Feruglio et al. 2010; Aalto et al. 2012; Cicone et al. 2014; García-Burillo et al. 2014). This has important implications for the co-evolution of galaxies and black holes which is observed through the now well-established $M_{\mathrm{BH}^{-}} \sigma$ relation (e.g., Gültekin et al. 2009).

Active galactic nuclei are observed in two categories, type 1 with broad-line regions (BLR) and type 2 with only 
narrow-line regions (NLR). Lines are broad only very close to the black hole, in the accretion disk, while they are narrow farther out in the NLR of $\sim 0.1-1 \mathrm{kpc}$ size. The original unification paradigm proposes that the BLR in type 2 is obscured by a dusty molecular torus, along the line of sight of the observer (e.g., Antonucci \& Miller 1985; Urry \& Padovani 1995). However, since this early work many observations have shown that inclination and obscuration are not the only parameters distinguishing types 1 and 2; some of these types are intrinsically different and/or nuclear starbursts are confusing the picture (e.g., Imanishi \& Wada 2004; Hatziminaoglou et al. 2009). A strong challenge of the unification paradigm also comes from AGN changing look from type 1 to type 2 and vice versa, without evidence of variable obscuration (e.g., LaMassa et al. 2015; McElroy et al. 2016).

The expected torus is so small $(3-30 \mathrm{pc}$ in size) that it was not possible to resolve it until recently, where $\mathrm{CO}(6-5)$ emission was detected for the first time with ALMA, as well as continuum and dense gas tracers, in a $10 \mathrm{pc}$-diameter torus in the Seyfert 2 NGC 1068 (García-Burillo et al. 2016; Gallimore et al. 2016; Imanishi et al. 2016, 2018). Dusty tori have also been seen in the near or mid-infrared (e.g., Asmus et al. 2011; Gratadour et al. 2015), although sometimes the dust emission is seen in the polar direction instead (Asmus et al. 2016).

García-Burillo et al. (2016) have obtained a high-resolution map in $\mathrm{CO}(6-5)$ with ALMA towards the center of NGC 1068: there is a circumnuclear disk (CND) $\sim 300 \mathrm{pc}$ in size, which is also detected in dust continuum. The AGN is offset with respect to the center of this disk. Around the AGN, a peak of CO emission is detected. This is identified as the molecular torus surrounding the AGN. The dust emission coincides spatially with the molecular torus. Two components were revealed, a dust torus $\sim 7 \mathrm{pc}$ in diameter, oriented along $\mathrm{PA}=142^{\circ}$, aligned with the $\mathrm{H}_{2} \mathrm{O}$ maser disk (Greenhill et al. 1996) and some polar emission that extends $10 \mathrm{pc}$ to the $\mathrm{SW}$. The $\mathrm{CO}(6-5)$ torus is a bit larger in size (diameter $\sim 10 \mathrm{pc}$ ) than the dust torus, and it appears tilted, with a $\mathrm{PA}=112^{\circ}$ with respect to the dust torus and accretion disk. The extent of the torus depends slightly on its tracer; it is somewhat larger with low-J CO emission (García-Burillo et al., in prep.) or in dense gas tracers (Imanishi et al. 2018). There is no CO counterpart for the polar emission. The molecular torus reveals strong non-circular motions and a large degree of turbulence. It also appears more face-on at larger radii, being probably warped. The perturbations in the morphology and kinematics of the torus can be interpreted in terms of the Papaloizou \& Pringle (1984) instability (PPI), predicted in particular for the dynamical evolution of AGN tori.

The discovery of such a perturbed and turbulent torus was a surprise. Is it due to continuous accretion from the CND onto the torus, triggering tilt, warp, and PPI instabilities, and finally leading to the AGN fueling? How does the molecular outflow detected farther away (García-Burillo et al. 2014) arise from the torus? Are perturbed and turbulent tori the norm in AGNdominated galaxies?

In the present paper, we describe our effort to gather more information on possible molecular tori in nearby Seyfert galaxies. We have observed with high spatial resolution seven barred galaxies with active nuclei in order to explore both the AGN fueling and the feedback processes, and also to characterize the molecular content inside the central kpc at 4-9 pc resolution. In many cases the gas kinematics allows us to refine the determination of the central black hole mass. Section 2 presents the galaxy sample, and Sect. 3 the details of the ALMA observations. The results are then described in Sect. 4 with first a brief discussion of the

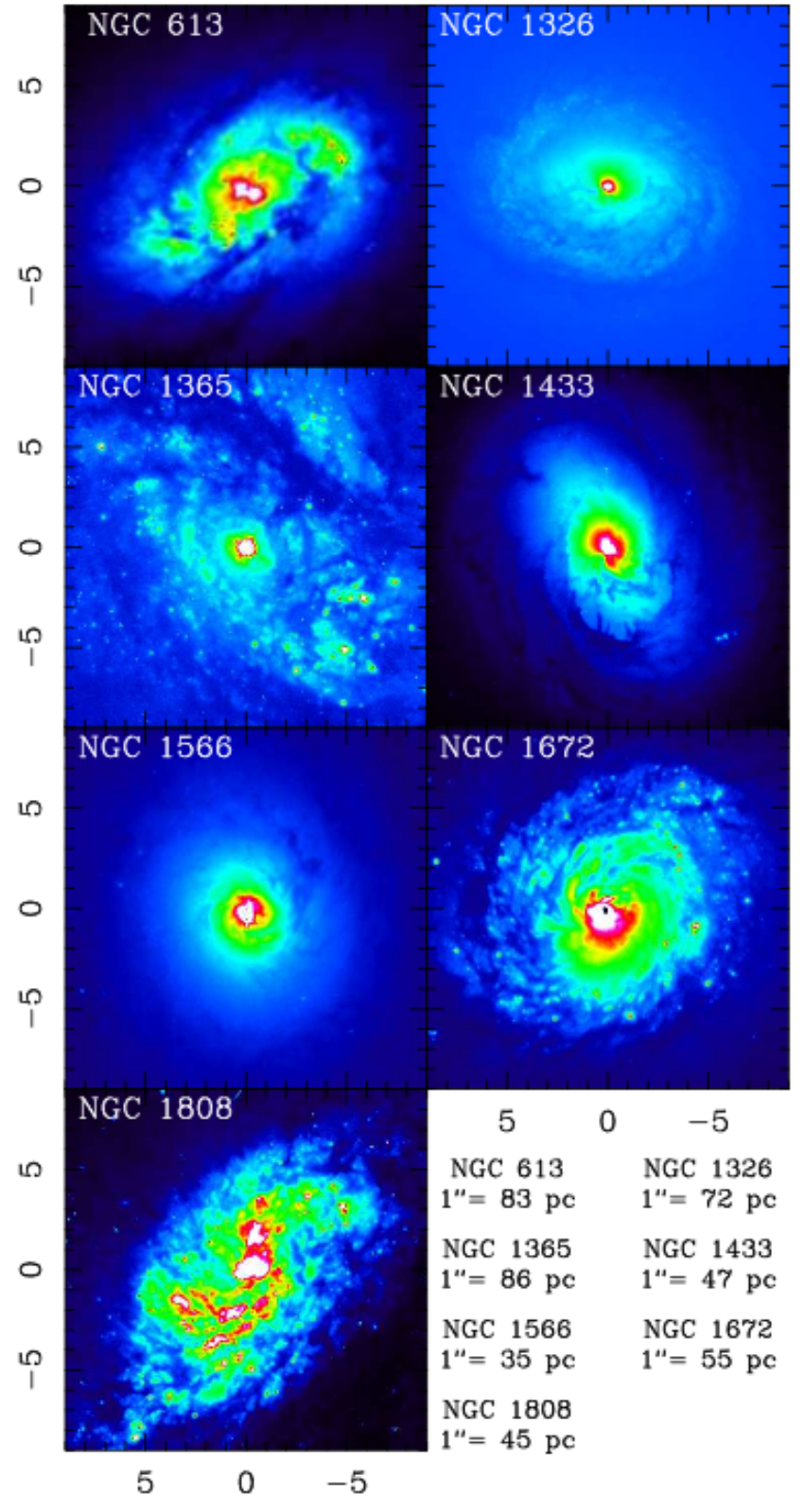

Fig. 1. Red HST images (F814W) for the seven sample galaxies in the same FOV $=18^{\prime \prime}$ as obtained with ALMA in Band 7. The axis labels correspond to arcsec, with north up and east to the left.

main features discovered, whether there is a molecular torus, the determination of its properties, and then the estimation of black hole masses. Section 5 summarizes and discusses our findings.

\section{The sample}

In addition to NGC 1433 and 1566 (Combes et al. 2013, 2014), we selected the five nearby southern AGN galaxies for which we previously gathered $\mathrm{CO}(3-2)$ observations at $0.14^{\prime \prime}$ resolution. They span more than a factor of 100 in AGN power (X-ray and radio luminosities), a factor of 10 in star formation rate (SFR), and a wide range of galaxy inner morphology (with or without double bars). This sample has been selected to provide a wide range of gas inflow rate, AGN feeding rate, and therefore test the various possible phases of evolution for the molecular tori. The galaxies were also selected to be sufficiently nearby to allow ALMA, with its exquisite spatial resolution, to 
resolve the torus if present. All galaxies have single-dish millimeter data with the SEST (see references in Table 1), and we have obtained ALMA cycle 0 or 3 intermediate resolution data, in addition to the present high-resolution ALMA cycle 3-4 CO maps. Some of the targets have also been observed by previous authors with ALMA at lower resolution of 50-100 pc (e.g., for NGC 613 and NGC 1808 Miyamoto et al. 2017; Salak et al. 2016, 2017). We used these observations in the archive when available. Our targets have high-resolution Hubble Space Telescope (HST) images, and are found in the IRAS Bright Galaxy Sample (Sanders et al. 2003). Most of these galaxies have been searched for $\mathrm{H}_{2} \mathrm{O}$ masers (tracing the accretion disk) with a 2/7 detection rate (Zhang et al. 2012; Surcis et al. 2009) and for starforming ("active") inner rings by Comerón (2013). In addition, we have proprietary SINFONI IFU observations of all galaxies, so that we can compare warm $\mathrm{H}_{2}$ morphology, ionized gas distributions, and kinematics with the cold molecular counterparts. We summarize the galaxy properties in Table 1, and the nuclear morphologies in Fig. 1.

\section{Observations}

To explore and characterize molecular tori in nearby Seyferts, we targeted the $\mathrm{CO}(3-2)$ line. For this transition, the $\mathrm{J}$ is low enough that it is still a tracer of density and mass more than excitation; it also has a relatively high flux and affords a high spatial resolution. It appears as the best compromise between spatial resolution, sensitivity, and field of view. To estimate $\mathrm{H}_{2}$ column densities and masses, we use in Sect. 4.3 the usual ratios applicable to active galaxy centers. The detailed study by Papadopoulos et al. (2012) has shown that the excitation ratios begin to depart from common ones at $J=5$ and beyond, and mainly for starbursts.

The observations were carried out with the ALMA telescope in cycles 3 and 4, with 36 to 40 antennas, during the years 2016 and 2017. The corresponding ALMA projects ID were \#2015.1.00404.S and \#2016.1.00296.S, both with PI F. Combes. In cycle 3, five galaxies (NGC 613, NGC 1326, NGC 1365, NGC 1672, and NGC 1808) were observed simultaneously in $\mathrm{CO}(3-2), \mathrm{HCO}^{+}(4-3), \mathrm{HCN}(4-3)$, and continuum, with Band 7. The compact configuration (TC, baselines 15 to $630 \mathrm{~m}$ ) and extended (TE, baselines 15 to $1400 \mathrm{~m}$ ) combined to give a synthesized beam of 0 .' $^{-14}(\sim 15 \mathrm{pc})$, and an rms sensitivity of $1 \mathrm{mJy} \mathrm{beam}^{-1}$ in $10 \mathrm{~km} \mathrm{~s}^{-1}$ channels $\left(80 \mu \mathrm{Jy} \mathrm{beam}^{-1}\right.$ in the continuum). The total integration time, including calibration and overheads, was $1 \mathrm{~h}$ per source. This choice of correlator configuration, selected to simultaneously observe three lines, provided a velocity range of $1600 \mathrm{~km} \mathrm{~s}^{-1}$ for each line. However, the various lines are not centered, and in particular a compromise had to be made for the $\mathrm{CO}(3-2)$ and $\mathrm{HCN}(4-3)$. These two lines are sampled with only $200 \mathrm{~km} \mathrm{~s}^{-1}$ on one side and $1400 \mathrm{~km} \mathrm{~s}^{-1}$ on the other. This is adequate for nearly face-on galaxies, but prevents seeing outflows on one side. The bandwidth was $1800 \mathrm{MHz}$ for the continuum bands.

In cycle 4 , the seven galaxies were observed at higher spatial resolution, $\sim 0$.' $^{\prime} 07$ or 4 to $9 \mathrm{pc}$ (depending on the various distances), to search for molecular tori. For the frequency tuning we chose to observe the $\mathrm{CO}(3-2)$ and the $\mathrm{HCO}^{+}(4-3)$ lines, and the continuum in Band 7. The HCN(4-3) transition was not observed in order to avoid a restricted velocity range in the expected broader spectral lines towards the nuclei. The observations were done in several blocks, a compact configuration (TM2, baselines 19 to $500 \mathrm{~m}$ ) and extended (TM1, baselines 19 to $3100 \mathrm{~m}$ ), with a total duration of two hours per galaxy. When combining two or more of these configurations, all calibrated observations with all baselines were included to obtain the UV-tables in CASA or GILDAS. The sensitivity reached was between 0.6 and $0.8 \mathrm{mJy}$ in $10 \mathrm{~km} \mathrm{~s}^{-1}$ channels. A summary of all configurations observed is given in Table 2.

The observations were all centered on the nuclei, with a single pointing covering a field of view (FOV) of $18^{\prime \prime}$. For NGC 1365, due to the large size of the galaxy and nuclear ring and the strength of $\mathrm{CO}$ emission, we performed a rectangular 13-point mosaic, ensuring a FOV of $45 \times 36^{\prime \prime}$, aligned on the major axis. The galaxies were observed in dual polarization mode with $1.875 \mathrm{GHz}$ total bandwidth per baseband, and a channel spacing of $0.488 \mathrm{MHz}$ corresponding to $\sim 0.8 \mathrm{~km} \mathrm{~s}^{-1}$, after Hanning smoothing. The spectra were then smoothed to 11.7 MHz $\left(10.2 \mathrm{~km} \mathrm{~s}^{-1}\right)$ to build channel maps.

The total integration time provided an rms of $30 \mu \mathrm{Jy}$ beam $^{-1}$ in the continuum, and $\sim 0.6 \mathrm{mJy}^{\text {beam }}{ }^{-1}$ in the line channel maps (corresponding to $\sim 1.2 \mathrm{~K}$ at the obtained spatial resolution). The flux calibration was done with nearby quasars, which are regularly monitored at ALMA, and resulted in 10\% accuracy.

The data were calibrated, imaged, and cleaned with the CASA software (versions 4.5.3 to 4.7.2; McMullin et al. 2007) and the analysis was then finalized with the GILDAS software (Guilloteau \& Lucas 2000). The final cubes at high resolution are at the maximum $1800 \times 1800$ pixels with 0 .' $^{\prime} 01$ per pixel in the plane of the sky, and have 60 channels of $10 \mathrm{~km} \mathrm{~s}^{-1}$ width. The maps were made with Briggs weighting and a robustness parameter of 0.5 , i.e., a trade-off between uniform and natural weighting. The data were cleaned using a mask made from the integrated $\mathrm{CO}(3-2)$ map. The continuum was subtracted from all line maps.

The final maps were corrected for primary beam attenuation. Very little $\mathrm{CO}(3-2)$ emission was detected outside the full width half power (FWHP) primary beam. Because of missing short spacings, the interferometer plays the role of a high-frequency filter, insensitive to smooth and extended emission; the scales that might be filtered out are those larger than $\sim 3^{\prime \prime}$, in each channel map. Since the velocity gradients are high in galaxy nuclei, this does not affect the line measurements too much; indeed, the size in each velocity channel is not expected to be extended. However, the missing-flux problem might affect continuum maps. Low-level negative sidelobes adjacent to bright emission were observed.

The exact values of missing flux amount will be detailed in forthcoming papers. We estimated the total $\mathrm{CO}(3-2)$ line flux for the two galaxies previously observed in cycle 0 (with the most compact configuration) to give an order of magnitude (see Table 3). The difference between the cycle 0 observations with beams of 0.5 and the combination TM1+TM2 shows that we are missing $\sim 25 \%$ of the total flux, on large angular scales. This will affect only slightly the masses estimated for the tori since they are not extended, except for NGC 1566. In future papers, all configurations will be combined to minimize flux losses.

\section{Results}

Figures 2-5 display the $\mathrm{CO}(3-2)$ flux distribution (moment 0 ) together with the velocity field (moment 1 ) of the seven targets; they demonstrate the wide variety of morphologies encountered. There are both resonant rings and spiral arms, and six galaxies host a molecular torus, either nearly face-on or more edge-on. We define a torus by the smallest decoupled circumnuclear structure in the $\mathrm{CO}(3-2)$ line, and from its morphology and kinematics. The morphology decoupling means that a nuclear disk or ring is clearly detached from the outside spiral structure or outer rings, and the corresponding kinematic displays 
Table 1. Characteristics of the sample.

\begin{tabular}{lcccccccccc}
\hline \hline Name & Type & $\begin{array}{c}\mathrm{D} \\
\mathrm{Mpc}\end{array}$ & $\begin{array}{c}\mathrm{SFR} \\
M_{\odot} \mathrm{yr}^{-1}\end{array}$ & $\begin{array}{c}\log \left(L_{X}\right) \\
\mathrm{erg} \mathrm{s}^{-1}\end{array}$ & $\begin{array}{c}\log \left(L_{1.4 \mathrm{GHz}}\right) \\
\mathrm{W} \mathrm{Hz}^{-1}\end{array}$ & $\begin{array}{c}\mathrm{S}(\mathrm{CO})_{21} \\
\mathrm{Jy} \mathrm{km} \mathrm{s}^{-1}\end{array}$ & $\begin{array}{c}\text { Bar } \\
\text { PA }\left({ }^{\circ}\right)\end{array}$ & $\begin{array}{c}\text { Double } \\
\text { bar? }\end{array}$ & $\begin{array}{c}\text { RA } \\
\text { ICRS }\end{array}$ & $\begin{array}{c}\text { Dec } \\
\text { ICRS }\end{array}$ \\
\hline N613 & Sy- SB(rs)bc & 17.2 & $5.3^{*}$ & 41.2 & 21.8 & $1200^{a}$ & 127 & 122 & $01: 34: 18.189$ & $-29: 25: 06.59$ \\
N1326 & LINER- SB0(r) & 14.9 & 1.1 & 39.9 & 20.9 & $200^{b}$ & 30 & 90 & $03: 23: 56.416$ & $-36: 27: 52.68$ \\
N1365 & Sy 1.8- SB(s)b & 17.8 & 17. & 41.8 & 22.3 & $2300^{c}$ & 92 & 46 & $03: 33: 36.368$ & $-36: 08: 25.51$ \\
N1433 & Sy 2- SB(r)ab & 9.7 & 0.5 & 39.2 & 20.0 & $360^{a}$ & 95 & 32 & $03: 42: 01.49$ & $-47: 13: 20.2$ \\
N1566 & Sy 1.5- SAB(s)bc & 7.2 & 0.8 & 40.5 & 21.3 & $540^{a}$ & 5 & No & $04: 20: 00.395$ & $-54: 56: 16.61$ \\
N1672 & Sy 2- SB(s)b & 11.4 & 3.1 & 38.4 & 19.9 & $1140^{a}$ & 97 & No & $04: 45: 42.496$ & $-59: 14: 49.91$ \\
N1808 & Sy 2- SAB(s)a & 9.3 & 4.7 & 39.8 & 21.7 & $4500^{d}$ & 139 & 158 & $05: 07: 42.329$ & $-37: 30: 45.85$ \\
\hline
\end{tabular}

Notes. D are the median values of $z$-independent distances from NED (Steer et al. 2017). SFR are derived from infrared luminosities (NED). ${ }^{(*)} \mathrm{NGC} 613$ has an $\mathrm{H}_{2} \mathrm{O}$ maser. $L_{X}$ is from 2-10 keV INTEGRAL, Rosat, and/or Chandra archives. The $\mathrm{CO}(2-1)$ integrated fluxes are from singledish measurements (SEST, beam 22") from ${ }^{(a)}$ Bajaja et al. (1995), ${ }^{(b)}$ Garcia-Barreto et al. (1991), ${ }^{(c)}$ Sandqvist (1999), ${ }^{(d)}$ Aalto et al. (1994). PA of bars are from Jungwiert et al. (1997) for NGC 613 and 1433, from Garcia-Barreto et al. (1991) for NGC 1326, Lindblad (1999) for NGC 1365, Agüero et al. (2004) for NGC 1566, Jenkins et al. (2011) for NGC 1672, and Dahlem et al. (1994) for NGC 1808. The RA-Dec positions are the new adopted centers for each galaxy, derived from the detected continuum point sources in the present work, with an error bar of $\sim 0.1^{\prime \prime}$ (Sect. 4.1).

Table 2. Configuration of the observations and resulting spatial resolution (with robustness parameter of 0.5 , see Sect. 3).

\begin{tabular}{lll}
\hline \hline Galaxy & Cycle 3, TC \& TE & Cycle 4, TM2 \& TM1 \\
\hline N 613 & $0.41 \times 0.35 \& 0.15 \times 0.13^{\prime \prime}$ & $0.33 \times 0.30 \& .092 \times .064^{\prime \prime}$ \\
N1326 & $0.35 \times 0.26 \& 0.16 \times 0.14^{\prime \prime}$ & $0.36 \times 0.29 \& .086 \times .058^{\prime \prime}$ \\
N1365 & $0.33 \times 0.24 \& 0.15 \times 0.14^{\prime \prime}$ & $0.35 \times 0.28 \& .087 \times .060^{\prime \prime}$ \\
N1433 & $0.56 \times 0.42^{\prime \prime} a$ & $0.37 \times 0.30 \& .067 \times .059^{\prime \prime}$ \\
N1566 & $0.64 \times 0.43^{\prime \prime} a$ & $0.35 \times 0.29 \& .061 \times .045^{\prime \prime}$ \\
N1672 & $0.35 \times 0.19 \& 0.16 \times 0.12^{\prime \prime}$ & $0.41 \times 0.29 \& .096 \times .064^{\prime \prime}$ \\
N1808 & $0.36 \times 0.24 \& 0.14 \times 0.11^{\prime \prime}$ & $0.30 \times 0.29 \& .082 \times .064^{\prime \prime}$ \\
\hline
\end{tabular}

Notes. ${ }^{(a)}$ Obtained in cycle 0 (Combes et al. 2013, 2014). TC and TM2 are compact configurations, TE and TM1 are extended.

a strong velocity gradient compared to the outside structure. In some cases the kinematic major axis of the inner torus is not aligned with the kinematic major axis of the outside structure (e.g., NGC 613, NGC 1566, NGC 1672 and NGC 1808). The $\mathrm{HCO}^{+}(4-3)$ line, when clearly detected, supports this definition (see Fig. 7). The dust continuum may also trace this circumnuclear structure, but it is weaker and tends to be less extended radially. Also, the central point source, coming in general from synchrotron AGN emission, perturbs its morphology. We emphasize that the radial extent of the torus may depend on the tracer considered, as already found in NGC 1068 (García-Burillo et al. 2016, and in prep.). Most of the time there are departures from symmetry, and the central torus is slightly offset from the barycenter of the $1 \mathrm{kpc}$-structure. In two cases (NGC 613 and NGC 1566) there is a clear trailing spiral inside the inner Lindblad resonance (ILR) ring, a "smoking gun" signature for ongoing fueling of the central black hole. The main morphological and kinematical features are described for each galaxy below.

\subsection{Continuum emission and AGN position}

All galaxies were observed with their phase center coinciding with the position given in NED. However, we now have a high spatial resolution, and when the nucleus has a strong continuum point source, it is possible to refine the position of the central black hole, expected to be the source of strong radio emission. A central continuum point source has been detected in all galaxies, except in NGC 1433 where we had a tentative detection in our
Table 3. Estimation of the missing $\mathrm{CO}(3-2)$ flux in the FOV of $18^{\prime \prime}$ for the two galaxies observed in cycle 0 .

\begin{tabular}{lccc}
\hline \hline Galaxy & Cycle 0 & TM2+TM1 & TM1 \\
\hline NGC 1433 & 234 & 174 & 161 \\
NGC 1566 & 596 & 402 & 364 \\
\hline
\end{tabular}

Notes. All integrated fluxes are in $\mathrm{Jy} \mathrm{km} \mathrm{s}^{-1}$.

previous work (Combes et al. 2013), but were not able to subsequently confirm it. We adopt, however, the same previously determined position for the center, which corresponds to the center of the inner nuclear ring. For all others, the center position adopted is the pixel of maximum continuum emission, determined with an error equal to the TM1 beam indicated in Table 2, typically $0.1^{\prime \prime}$. These positions are displayed in Table 1 , and in the following are considered to coincide with the actual AGN nucleus. These positions are now better determined than the optical nuclei defined by the maximum brightness in the HST images of Fig. 1, which have an astrometry accurate to within $\leq 1^{\prime \prime}$. Our adopted positions also coincide most of the time with the maximum velocity dispersion in the $\mathrm{CO}(3-2)$ line (except for NGC 1365 and NGC 1566).

There is also the possibility that some continuum emission from the unresolved central source is coming from the inner torus around the AGN. Most galaxies in the sample are low-luminosity AGN in which the dust emission close to the nucleus is expected to emerge between 10 and $300 \mu \mathrm{m}$ (Casey 2012). We expect that the continuum point source at $0.8 \mathrm{~mm}$ is dominated by synchrotron emission (Prieto et al. 2010). Around the unresolved continuum sources, Figs. 2-5 show that we detect extended emission which might be dominated by dust emission from the inner tori; however, these tori are better traced by the CO line emission.

\subsection{Molecular gas distribution and morphology}

NGC 613. shows an incomplete ring with high-density contrast, as seen in our large-scale map of $\mathrm{CO}(3-2)$ (Fig. 2), and emission from a clear trailing two-arm spiral structure in the circumnuclear disk (CND). This galaxy has a typical ILR nuclear ring, of radius $3.5 \mathrm{arcsec}$ (or $300 \mathrm{pc}$ ), just inside the two characteristic leading dust lanes of the bar, which are tangent to the 

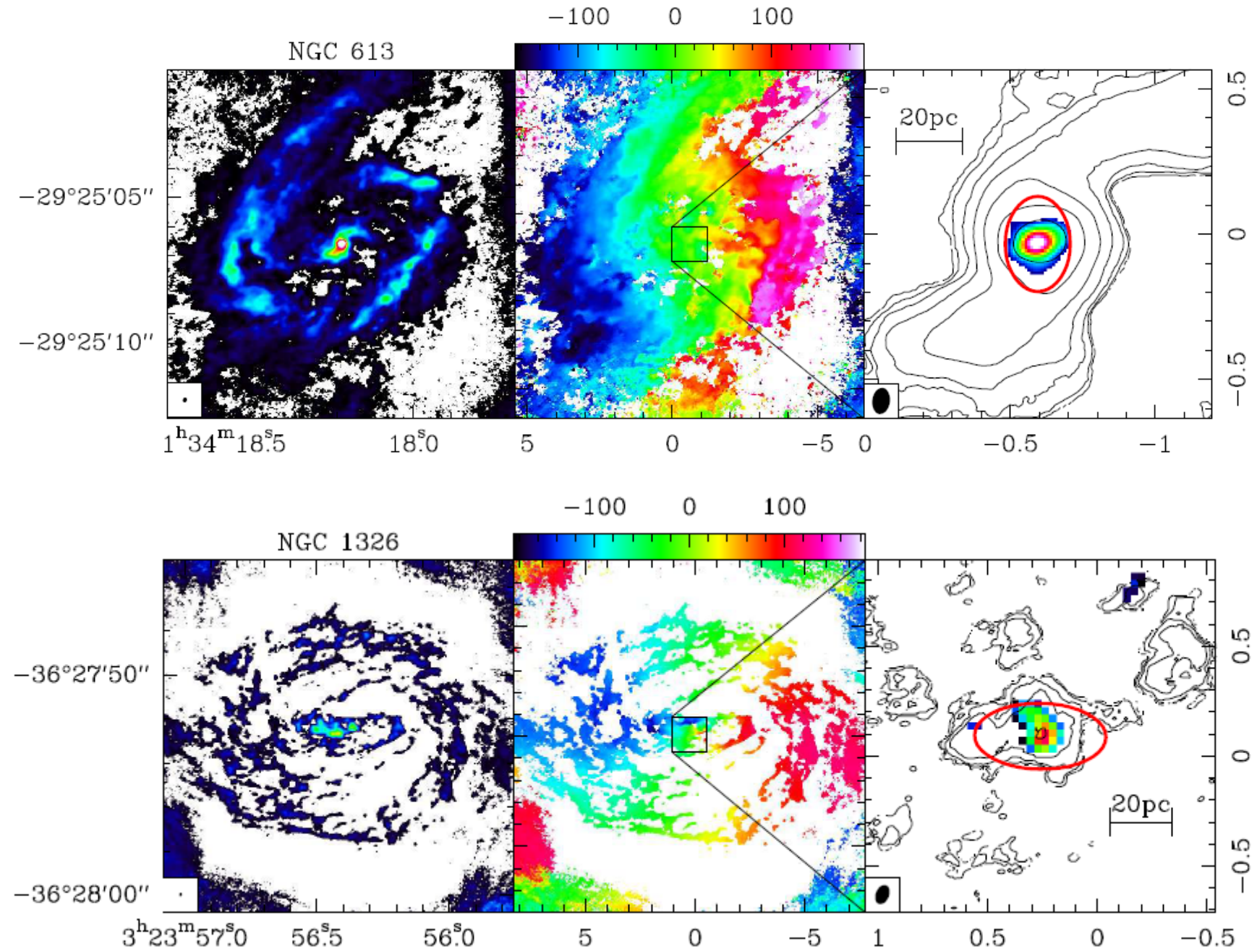

Fig. 2. Top panel: CO(3-2) moment 0 and 1 of galaxy NGC 613 (left and middle, TE+TM1). Right panel: zoom-in of the CO(3-2) contours (of the TM1 observations at high resolution) superposed on a color scale of the continuum emission in Band 7. The three panels have been centered on the central continuum source, and the labels are the offset in arcseconds from the phase center. For the two first panels the beam size is indicated at the bottom left. In the third panel is shown the ellipse (in red) used to define the molecular tori in Sect. 4.3. The contours are from $25 \sigma$ to $1600 \sigma$, and follow each other by factor 2 multiplication. The scale bar in the right panel is $20 \mathrm{pc}$ long. The color scale in the middle panel is in $\mathrm{km} \mathrm{s}^{-1}$. Bottom panel: same, but for galaxy NGC 1326. The contours are from $3 \sigma$ to $96 \sigma$, and follow each other by factor 2 multiplication.

ring. Inside the ring, there is a central molecular component, or CND of radius $\sim 1^{\prime \prime}=83 \mathrm{pc}$. Water masers have been detected in the nucleus (Kondratko et al. 2006). Miyamoto et al. (2017) have mapped the ring in $\mathrm{CO}(1-0)$ and $\mathrm{CO}(3-2)$ with ALMA at 0.7 and $0 . ' 4$, respectively, and found a clumpy ring, globally regular, but with spots of active and efficient star formation. ALMA finds a continuum jet at $95 \mathrm{GHz}$ with a $\mathrm{PA}=20^{\circ}$ (Miyamoto et al. 2017), which corresponds to the $4.9 \mathrm{GHz}$ and $14.9 \mathrm{GHz}$ jets (Hummel \& Jorsater 1992), close to the minor axis of the ring. At $350 \mathrm{GHz}$, the central continuum source remains unresolved at our resolution of 0.'09. The negative slope found for the flux over frequency $\alpha \sim 0.6$ is compatible with synchrotron emission, with a small fraction of free-free.

The ring reveals two breaks into two winding spiral arms to the north and south. The bar has a position angle $\mathrm{PA}=130^{\circ}$ and is delineated on its leading sides by two dust lanes: one runs from the SW of the nucleus to the east side of the bar $\left(\mathrm{PA}=130^{\circ}\right)$, and the other from the NE of the nucleus to the west side of the bar $\left(\mathrm{PA}=-50^{\circ}\right)$ (see Fig. 1). The spiral arms are the beginning of these characteristic dust lanes along the bar: they are the contact points between the tangent lanes and the ring.
Dense gas $\left(>10^{6} \mathrm{~cm}^{-3}\right)$ is detected in various lines of $\mathrm{HCN}$, $\mathrm{HCO}+, \mathrm{CS}$, and $\mathrm{SiO}$ at the edges of the jets (Miyamoto et al. 2017). We find that at high resolution with $\mathrm{HCO}^{+}$(4-3) (Fig. 7) the dense molecular gas is very concentrated towards the nucleus, with skewed kinematics, suggesting an outflow along the jets; this will be described in a future paper (Audibert et al., in prep.). A first look at it can be seen in Fig. 14, where both the velocity field and velocity dispersion are perturbed. The outflow has already been suggested by the high-velocity dispersion of the [FeII] line along the radio jet (Falcón-Barroso et al. 2014; Davies et al. 2017).

A coherent chronology of star formation possibly driven by the gas motions in the ring was sought by Böker et al. (2008) with SINFONI images in $\mathrm{Br} \gamma$, HeI, and [FeII] (spectral resolution $R=2000$, and without adaptive optics). They identified clumps along the ring, color-coded according to the different star formation phases. These authors searched for a coherent chronology of star formation, starting from the dust lanes, fueling gas into the ring. At least in the southern part of the ring the expected sequence of star formation was indeed observed: the hottest stars were found near the contact point 

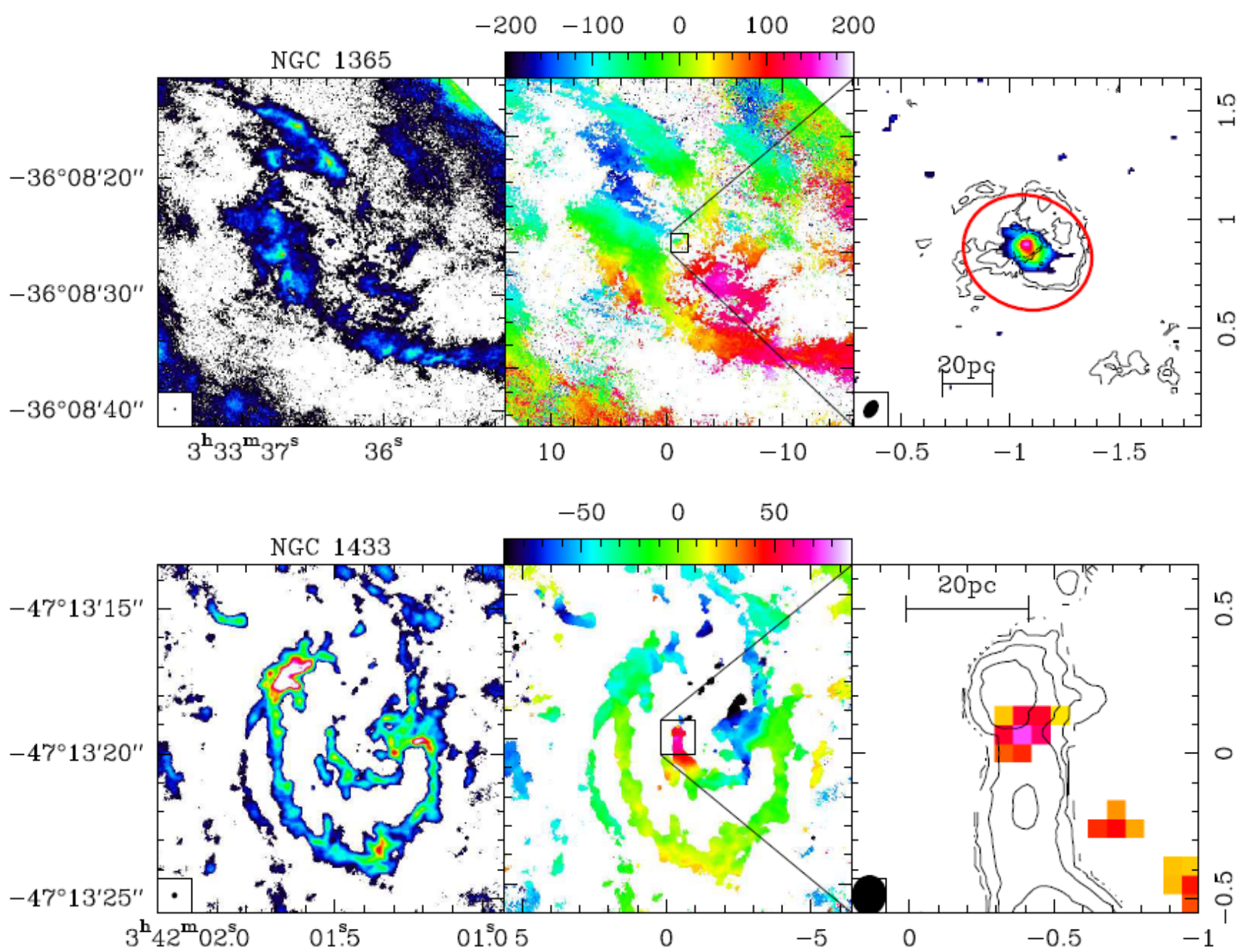

Fig. 3. Top panel: same as Fig. 2 for NGC 1365 (from the center of the mosaic; the size of the beam has been multiplied by 1.5 to become visible in the first panel) - the contours are from $4 \sigma$ to $256 \sigma$, and follow each other by factor 2 multiplication - and bottom panel: for NGC 1433 (in the latter case, TM2+TM1 in all 3 panels). The continuum emission (color plot in the right panel) maximizes at $0.2 \mathrm{mJy}$ (i.e., only slightly above $3 \sigma$ ) and was not detected in the TM1 configuration alone (detection only when combining TM1+TM2). The $\mathrm{CO}(3-2)$ line contours are from $3 \sigma$ to $192 \sigma$, and follow each other by factor 2 multiplication.

(defined above between the dust lane and the ring), and then fewer hot stars were found along the ring. The star formation scenario is not the random one, where new stars light up like a popcorn model. If gas is inflowing from the bar dust lanes into the ring, as expected from gravity torques (García-Burillo et al. 2005), there must also be an inflow in the CND due to the nuclear trailing spiral, as already observed and interpreted for NGC 1566 (Combes et al. 2014). The detailed nature of the gas flows in NGC 613 will be studied in a future work. Inside the nuclear spiral structure, there is a very dense and compact (radius $\sim 14 \mathrm{pc}$ ) rotating component, which might be interpreted as the molecular torus. The torus is also distinguished by a kinematic decoupling, with a major axis $\mathrm{PA}=0^{\circ}$, (better seen in $\mathrm{HCO}^{+}(4-3)$ ), while the outside axis is $\mathrm{PA}=105^{\circ}$. The excitation of the ionized gas in the torus is dominated by shocks (Davies et al. 2017).

NGC 1326. is a lenticular barred galaxy with a contrasted ring at ILR, of radius 5.7" $=410$ pc. Garcia-Barreto et al. (1991) detected radio continuum in the ring at 20,6 , and $2 \mathrm{~cm}$ with the VLA at $\sim 3^{\prime \prime}$ resolution, and also a molecular mass of $2.7 \times 10^{8} M_{\odot}$ from CO emission with the SEST-15m telescope. There is no evidence of a strong central continuum point source at centimeter wavelengths. At $350 \mathrm{GHz}$, we detect a weak con- tinuum source, coinciding with the maximum of the $\mathrm{CO}(3-2)$ emission (Fig. 2). About $83 \%$ of the $\mathrm{H} \alpha$ emission of the galaxy is coming from this nuclear ring (Crocker et al. 1996). There are no conspicuous dust lanes along the bar, and the ring is not interrupted by spiral structure at their contact. The ring in $\mathrm{CO}$ and also in $\mathrm{H} \alpha$ is oriented at $\mathrm{PA}=90^{\circ}$ roughly perpendicular to the bar $\left(\mathrm{PA}=20^{\circ}\right)$, suggesting either that the gas is on $\mathrm{x} 2$ orbits, or that it forms a decoupled nuclear ring, with a pattern speed higher than that of the primary bar. In any case, this means that the primary bar pattern speed is slow enough to allow the presence of two ILRs. When the rotation curve of the galaxy (Garcia-Barreto et al. 1991; Storchi-Bergmann et al. 1996) is considered, the ring corresponds to the inner ILR.

The HST image in $\mathrm{H} \alpha$ (Buta et al. 2000) reveals a marked asymmetry of the ring, which we do not see in $\mathrm{CO}(3-2)$ with ALMA. While the $14^{\prime \prime}$-diameter $(1 \mathrm{kpc})$ ring overlaps very well in $\mathrm{H} \alpha$ and $\mathrm{CO}(3-2)$ emissions, it is depleted on its west side only in $\mathrm{H} \alpha$. This must therefore be due to strong dust extinction in the west side. The ring is made of hundreds compact sources, which could be star clusters, and also some diffuse emission, tightly wrapped and spiral in character. This spiral structure is seen in our $\mathrm{CO}(3-2)$ high-resolution map as 

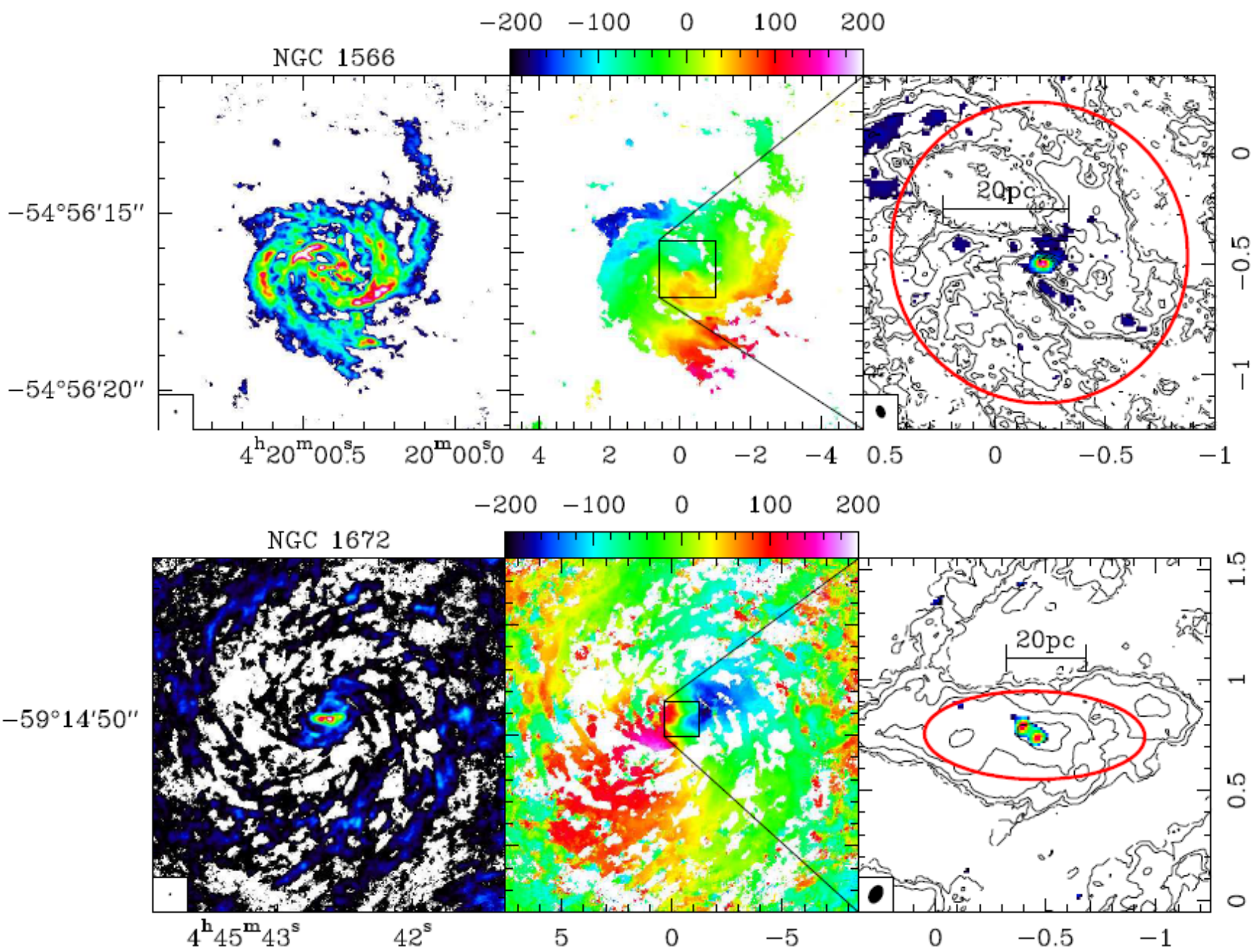

Fig. 4. Top panel: same as Fig. 2, but for NGC 1566 (in this case TM2+TM1 instead of TE+TM1 in the first two panels). The contours are from $5 \sigma$ to $160 \sigma$, and follow each other by factor 2 multiplication. Bottom panel: same, but for NGC 1672 . The contours are from $3 \sigma$ to $192 \sigma$, and follow each other by factor 2 multiplication.

well. Stellar populations were studied from U,B,V, I colors and $\mathrm{H} \alpha$-filter images by Buta et al. (2000). The age range of the stellar population in the ring is $10-200 \mathrm{Myr}$ and the derived star formation rate is $1 M_{\odot} / \mathrm{yr}$ (Buta et al. 2000). Inside the nuclear ring, the $\mathrm{CO}$ emission reveals a central $\mathrm{CND}$, of radius $\sim 0.3^{\prime \prime}=21 \mathrm{pc}$. This central structure appears slightly more inclined on the sky than the main galaxy, and is likely a tilted torus.

NGC 1365. is a strongly barred spiral galaxy with the characteristic dust lanes delineating the bar, which are particularly contrasted and curved. As an archetypal barred galaxy, it has been extensively observed (e.g., Lindblad 1999). While the Seyfert 1.8 nucleus is obvious in optical and X-rays (Nardini et al. 2015), it is hardly seen in radio. Stevens et al. (1999) discuss a marginal radio jet, the center of which corresponds to the central X-ray position.

The main part of the radio emission at centimeter wavelengths is the nuclear ring, with hot spots corresponding to star formation. Sakamoto et al. (2007) have mapped in $\mathrm{CO}(2-1)$ and isotopes a mosaic with SMA at 2 " resolution, that shows clearly a $2 \mathrm{kpc}$-extent oval ring, connected to the leading dust lanes, characteristics of the bar morphology. The AGN has only a small contribution $(\sim 5 \%)$ to the central infrared emission, which is dominated by star formation (Alonso-Herrero et al. 2012).
At $350 \mathrm{GHz}$, we detect a central continuum point source, as can be seen in Fig. 3. Our large-scale $\mathrm{CO}(3-2)$ map reveals the contrasted nuclear ring of radius $\sim 9^{\prime \prime}=770 \mathrm{pc}$. Inside this ring, which corresponds to the ILR of the bar, we detect a more compact molecular component, a CND with a ring shape, of radius $0.3=26 \mathrm{pc}$. This rotating ring just encircles the central continuum source, and might be interpreted as the molecular torus.

Lena et al. (2016) have recently presented optical integral field spectroscopy for the inner $6^{\prime \prime}$. They find evidence for a fanshaped blueshifted outflow in [NII] and $\mathrm{H} \alpha$ kinematics, corresponding to the outflow in a cone seen with the [OIII] emission lines, extending at more than $1 \mathrm{kpc}$ from the center along the minor axis (Hjelm \& Lindblad 1996; Venturi et al. 2017). From gravity torques, it was possible to show that the gas is inflowing to the center, driven by the bar, on a timescale of $300 \mathrm{Myr}$ (Tabatabaei et al. 2013). We will discuss in a future paper the possibility of an outflow in the molecular component (Audibert et al., in prep.).

NGC 1433. is a strongly barred spiral galaxy, nicknamed the "Lord of Rings" because of the presence of clear nuclear, inner, and outer rings at the bar resonances (Buta \& Combes 1996). Our previous cycle 0 ALMA observations in Band 7, with 0.'5 resolution, have revealed that inside the nuclear ring at $\sim 400 \mathrm{pc}$, there is also an inner nuclear ring at $\sim 200 \mathrm{pc}$, corresponding to 


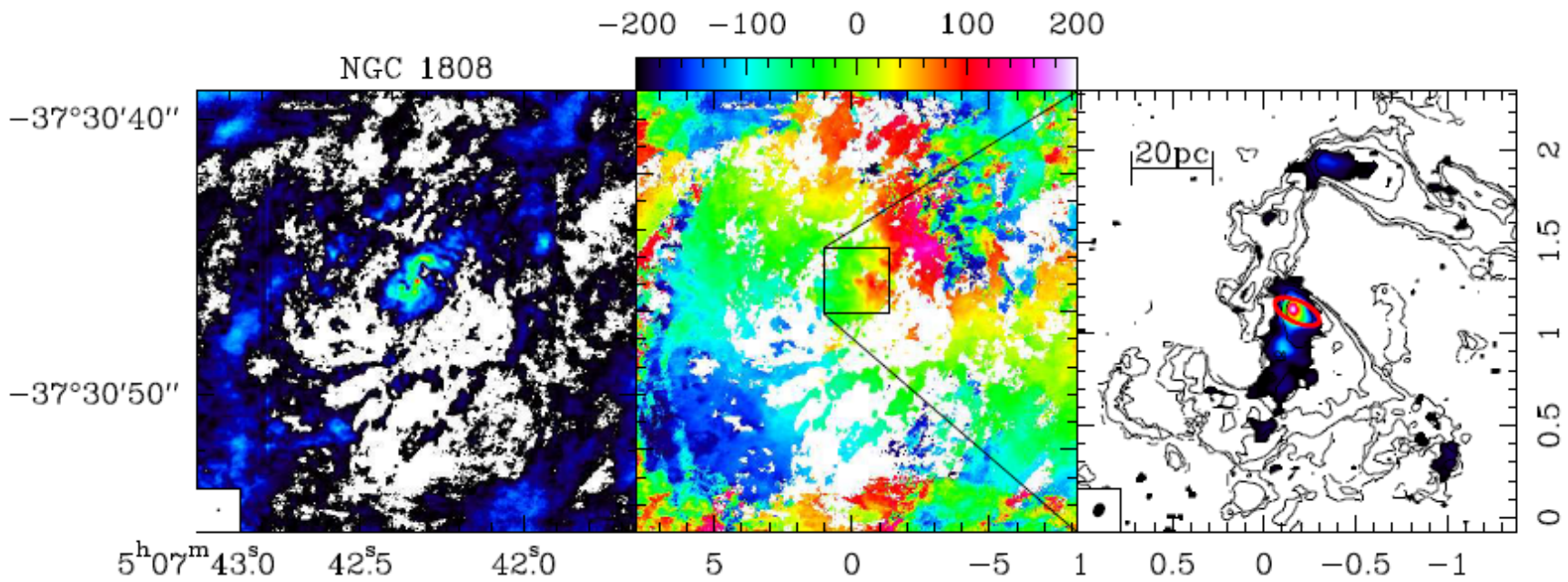

Fig. 5. Same as Fig. 2, but for NGC 1808. The contours are from $3 \sigma$ to $384 \sigma$, and follow each other by factor 2 multiplication. The color scale in the middle panel is in $\mathrm{km} \mathrm{s}^{-1}$.

the inner ILR (Combes et al. 2013). These observations revealed also a mild molecular outflow along the minor axis, of $7 M_{\odot} / y r$, the smallest molecular outflow ever observed in the Local Universe. It is possible that the outflow in a previous more powerful phase has destroyed a potential torus. The tentative continuum point source near the center is not confirmed, and there is no evidence of any molecular torus.

The only small velocity gradient in the center corresponds to the outflow along the minor axis. This is supported by the coincidence of the optical and near-infrared emission in the center, showing no extinction (Smajić et al. 2014). The CO(3-2) emission has a very filamentary structure at small scale, as can be seen in Fig. 3. The nuclear ring is the site of a starburst (Sánchez-Blázquez et al. 2011), and the gas is transiently stalled there though gravity torques (Smajić et al. 2014).

NGC 1566. is an intermediate barred spiral galaxy, possessing nuclear, inner and outer rings at resonances (Agüero et al. 2004). The broad lines detected in the nucleus, and the observed variability are typical of a Seyfert 1 (Alloin et al. 1985). It appears that this low-luminosity AGN appears has been increasing its activity over the last few hundred years; the line excitation is much higher in the BLR than along the NLR cone (Baribaud et al. 1992; Reunanen et al. 2002; Smajić et al. 2015).

Our previous cycle 0 ALMA observations have shown that the molecular gas followed a trailing spiral inside the nuclear ring, fueling the central black hole. The gravity torques due to the bar on the gas change sign at each resonance, and also change sign as the winding of spirals change from trailing to leading (Buta \& Combes 1996). When the spiral is leading inside the ring located at the inner Lindblad resonance, the torques are positive and the gas inside the ring is driven back to the ring. Instead, when the spiral is trailing, the torques are negative, the gas loses angular momentum, and is driven towards the nucleus. The sense of winding of the spiral must be due to the gravitational influence of the black hole itself (Combes et al. 2014; Smajić et al. 2015). The image at high resolution confirms the trailing spiral structure in the nuclear disk (Fig. 4). The nucleus is also the site of young star formation, and of a consequent velocity dispersion drop, also called $\sigma$-drop (da Silva et al. 2017; Emsellem et al. 2001).

Inside the inner spiral fueling the nucleus, there is a ringed structure of $0.7^{\prime \prime}=24 \mathrm{pc}$ radius, kinematically decoupled, which can be considered a molecular torus. As can be seen in Fig. 4, the torus kinematic major axis is vertical $\left(\mathrm{PA}=0^{\circ}\right)$, while the rest of the disk has a kinematic major axis $\mathrm{PA}=60^{\circ}$.

NGC 1672. is a strongly barred Seyfert 2 galaxy, with a high star-forming activity in its center. The AGN activity is therefore hard to find through line diagnostics (Storchi-Bergmann et al. 1996; Kewley et al. 2000). Brandt et al. (1996) with ROSAT find a diffuse X-ray nuclear source, rather soft, compatible with a superbubble interpretation (i.e., thermal emission from star formation). The star formation rate implies enough supernovae over a $10^{7} \mathrm{yr}$ period to blow a superbubble, as computed by Mac Low \& McCray (1988), although the X-ray gas pressure and density appear too high (Brandt et al. 1996). While finding two additional X-ray sources at the bar extremities, de Naray et al. (2000) conclude that the Seyfert 2 activity must be obscured by a Compton-thick nucleus with a column density of at least $10^{24} \mathrm{~cm}^{-2}$. The X-ray emission at the center appears diffuse and dominated by a starburst nucleus. Jenkins et al. (2011) with the high resolution of Chandra are the first to find a hard X-ray emission associated with the nucleus in addition to a ring. This confirms that the galaxy is actually a low-luminosity AGN, a Seyfert 2.

The circumnuclear ring, of $5^{\prime \prime}=275 \mathrm{pc}$ radius, is conspicuous in the radio emission at $3 \mathrm{~cm}$ from the VLA, and also in the Spitzer $8 \mu \mathrm{m}$ band, tracing essentially the PAH dust (Jenkins et al. 2011). Díaz et al. (1999) provide the H $\alpha$ velocity field in the central $2 \mathrm{kpc}$ : it has a strong velocity gradient, with a mass of $9 \times 10^{8} M_{\odot}$ inside $125 \mathrm{pc}$. The ring is located at the inner ILR, and is also quite contrasted in the $\mathrm{CO}(3-2)$ emission (Fig. 4). Inside the ring, some thin filaments join towards a central concentration, which looks like a torus of radius $0.5^{\prime \prime}=$ $27 \mathrm{pc}$ seen more inclined than the large-scale disk. A kinematic decoupling helps to define the torus, with a kinematic major axis of the torus of $\mathrm{PA}=90^{\circ}$, and an outside axis of $\mathrm{PA}=135^{\circ}$. The continuum emission peaks just at the center.

NGC 1808. is a barred starburst containing a Seyfert 2 nucleus. Optically, it is possible to see the dust lanes expelled perpendicular to the major axis. There is evidence of largescale (1 kpc) outflows, likely due to the starburst, and NGC 1808 has been classified as a superwind galaxy, similar to M 82 (Dahlem et al. 1990, 1994). The outflow is also seen in HI (Koribalski et al. 1993). Surprisingly, the outflow is not observed in the molecular component at the small scales sampled here 
(Audibert et al., in prep.). The outflow is thus apparently generated at a large distance from the nucleus $(>300 \mathrm{pc})$, favoring a starburst-driven flow over an AGN-driven one.

Star formation is very active through hot spots aligned on a ring of radius $10^{\prime \prime}=450 \mathrm{pc}$ (Koribalski et al. 1996). Inside this ring, also contrasted in CO (Salak et al. 2016), there is a circumnuclear disk or ring of radius $200 \mathrm{pc}$. Within this circumnuclear disk it is possible to see a trailing spiral in the $\mathrm{CO}$ (Fig. 5), which is even more contrasted in the dense gas tracers: $\mathrm{HCO}^{+}(4-3)$, $\mathrm{CS}$. The continuum is a point source inside the nuclear spiral, and may also correspond to a torus of radius $0.13^{\prime \prime}=6 \mathrm{pc}$. The previous ALMA observations by Salak et al. (2017) have shown the nuclear ring, and from the two contact points of the characteristic dust-lanes, two starting spiral arms. With a beam of $22^{\prime \prime} 6=100 \mathrm{pc}$, Salak et al. (2017) had insufficient spatial resolution to see the trailing spiral inside the nuclear ring. They call the nuclear ring a torus even if this component does not obscure the AGN. In the following, we will define the torus as the smaller compact structure of radius $6 \mathrm{pc}$ inside the nuclear spiral. The kinematic major axis of the torus is $\mathrm{PA}=100^{\circ}$, misaligned with the outside axis of $\mathrm{PA}=140^{\circ}$.

Busch et al. (2017) have published a near-infrared IFU spectroscopy of the inner $600 \mathrm{pc}$ with SINFONI in seeing-limited mode and $R=1500-4000$ spectral resolution. They determine an indicative black hole mass of $10^{7} M_{\odot}$. The age of the stars on the ring is homogeneous and younger than $10 \mathrm{Myr}$. They find shocked $\mathrm{H}_{2}$ warm gas near the nucleus, with non-circular motions. Although there is much gas streaming inside a radius of $1^{\prime \prime}=45 \mathrm{pc}$, there is no strong sign of nuclear activity.

\subsection{Masses of possible tori}

In at least six of the seven galaxies, there is evidence of a compact central ring or disk in $\mathrm{CO}$ emission, which can be interpreted as a molecular torus. These structures can be seen in $\mathrm{CO}(3-2)$ contours overlaid over HST images in Fig. 6. Their kinematics are also displayed with models, starting from Fig. 11. For NGC 1433, there is no evidence of a nuclear disk rotating around the nucleus, although there is a piece of spiral arm superposed on it. The molecular nuclear disks or tori are clearly decoupled from the rest of the disks in the kinematics as well. This is seen in Figs. 2-5, and also particularly in the dense gas maps in $\mathrm{HCO}^{+}$(4-3) which will be discussed in future papers. The example of NGC 613 is displayed in Fig. 7. In all galaxies, except for NGC 613 and NGC 1808, the size of the torus is much larger than the beam size (8-26 times). For NGC 613 and NGC 1808, the torus is only 4 times the beam, and the size reported in Table 4 has been deconvolved from the beam.

We have identified in the $\mathrm{CO}(3-2)$ data cube these components through ellipse-fitting with the adopted center as the continuum point source identified with the nucleus (at the coordinates given in Table 1). The sizes of the tori are illustrated by red ellipses in Fig. 2 and beyond. From the integrated flux $\mathrm{S}(\mathrm{CO}) \mathrm{dV}\left(\mathrm{Jy} \mathrm{km} \mathrm{s}^{-1}\right)$ found within the region, the derived molecular mass is obtained through the formula

$\mathrm{L}^{\prime}{ }_{\mathrm{CO}}\left(\mathrm{K} \mathrm{km} \mathrm{s} \mathrm{pc}^{2}\right)=3.25 \times 10^{7} \frac{\mathrm{S}(\mathrm{CO}) \mathrm{dV}}{(1+z)}\left(\frac{D_{\mathrm{L}}}{v_{\text {rest }}}\right)^{2}$,

where $v_{\text {rest }}=345.796 \mathrm{GHz}$, and $D_{\mathrm{L}}$ is the luminosity distance in Mpc (e.g., Solomon \& Vanden Bout 2005). The molecular mass, including helium, is then derived from

$\mathrm{M}\left(\mathrm{H}_{2}\right)=4.36 \mathrm{~L}^{\prime} \mathrm{CO} R_{13}$

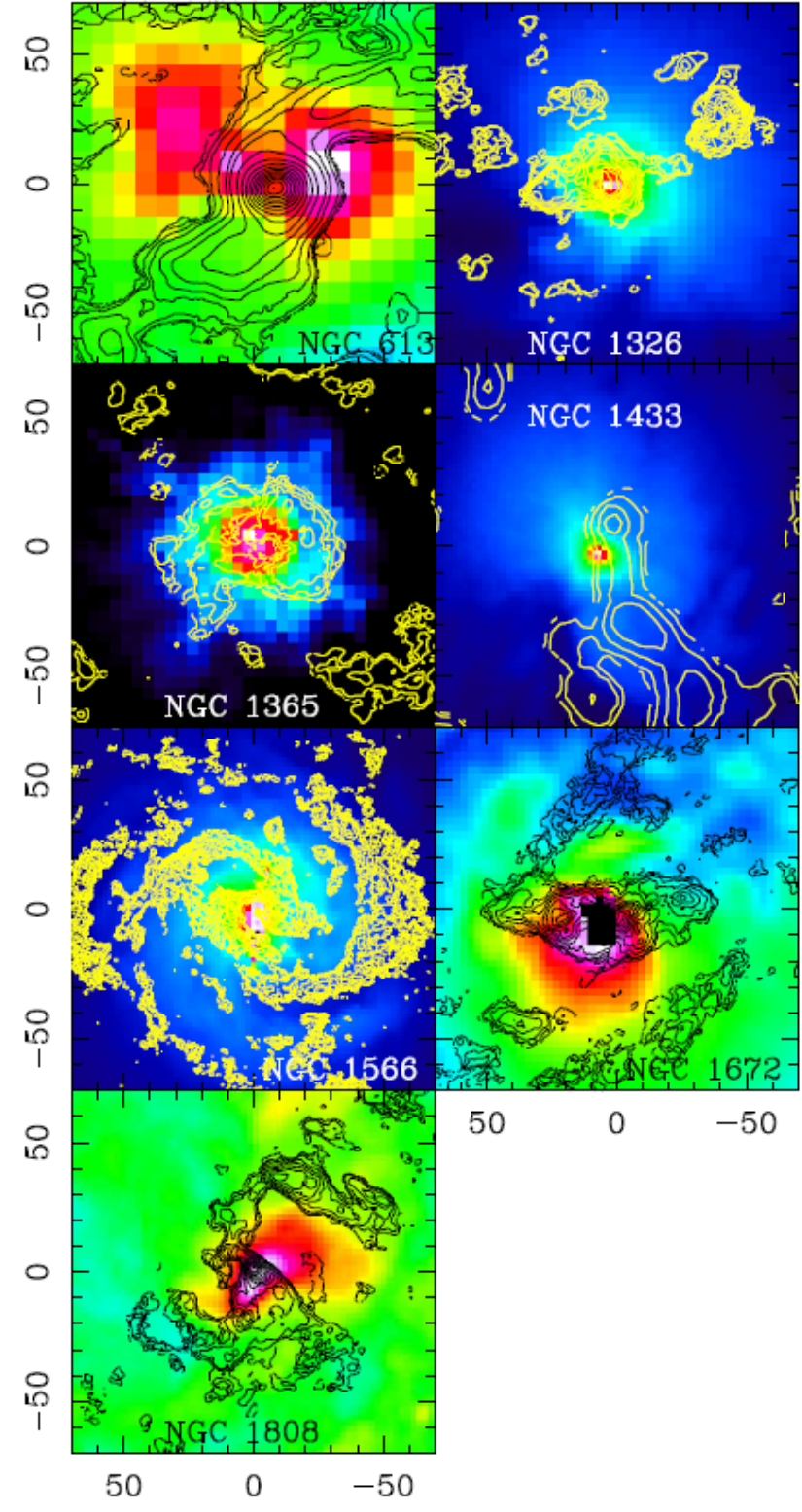

Fig. 6. Red HST images (F814W) for the seven sample galaxies, zoomed into the central field of $70 \mathrm{pc}$ radius, with the $\mathrm{CO}(3-2)$ contours overlaid. The axis labels are in pc.

assuming the standard CO-to- $\mathrm{H}_{2}$ conversion factor of $X_{\mathrm{CO}}=2 \times 10^{20} \mathrm{~cm}^{-2}\left(\mathrm{~K} \mathrm{~km} \mathrm{~s}^{-1}\right)$, applicable to Milky Waylike galaxies, and $R_{13}=L_{\mathrm{CO} 1-0}^{\prime} / L_{\mathrm{CO} 3-2}^{\prime}=2$ (e.g., Tacconi et al. 2013). Although this ratio was applied for high- $z$ galaxies, it should also be appropriate to local galaxy nuclei, where the molecular gas is dense (e.g., Braine \& Combes 1992; Dumke et al. 2001). The actual excitation of the molecular gas in the tori, close to the AGN, is yet not well known, and we refer to the above-mentioned papers, where our data on $\mathrm{HCN}, \mathrm{HCO}^{+}$, and CS lines are discussed.

We display in Table 4 the size and molecular masses of the tori. They vary from 6 to $27 \mathrm{pc}$ and 0.7 to $3.9 \times 10^{7} M_{\odot}$. In NGC 1326, 1672, and 1808, the torus is rather inclined on the sky plane, and could obscure the nucleus. This is well correlated with the Sy 2 type. The uncertainties on the torus size are determined from the extrema of the possible fits, taking into account both moments zero and one, and are never smaller than the beam size. The error bars on the integrated flux and other properties 


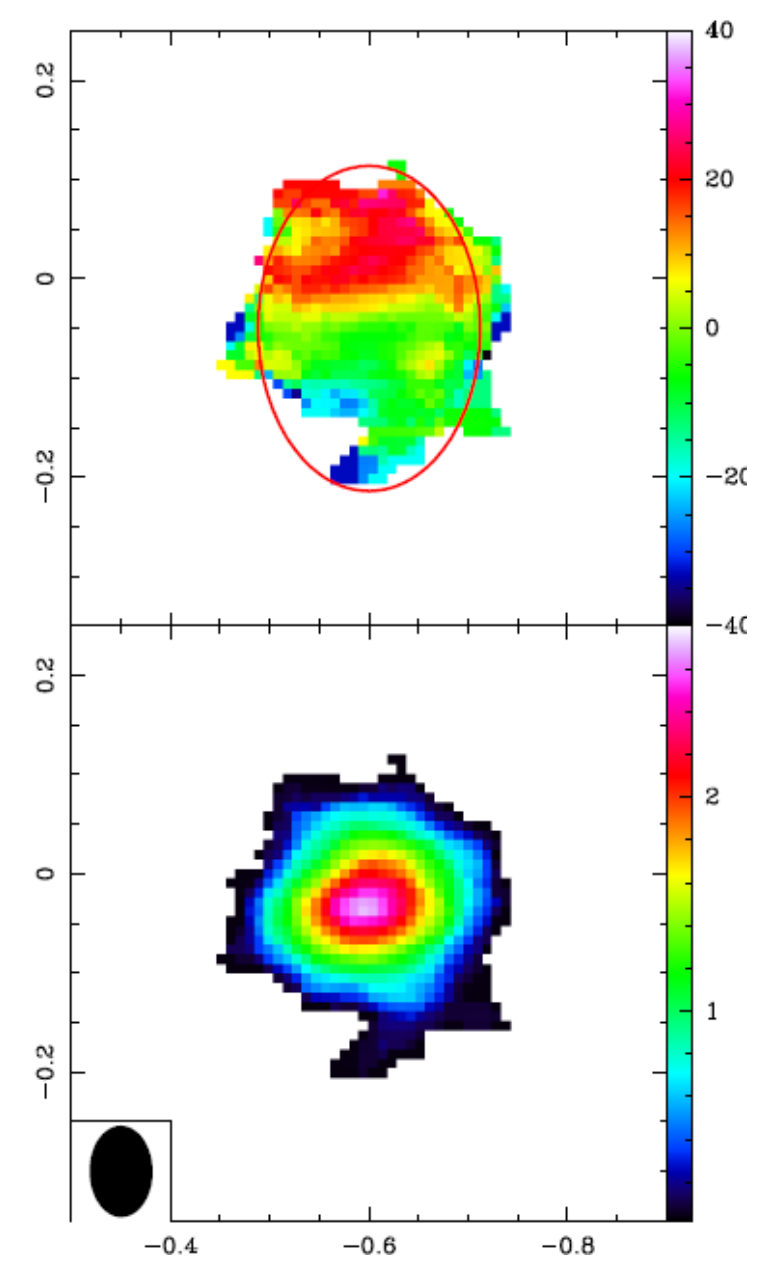

Fig. 7. First moments of the $\mathrm{HCO}^{+}(4-3)$ map of NGC 613. The color scale of the intensity at the bottom is in Jy beam ${ }^{-1} \times \mathrm{km} \mathrm{s}^{-1}$, and the velocity at the top is in $\mathrm{km} \mathrm{s}^{-1}$. The red ellipse at the top indicates the definition of the molecular torus. The RA-Dec scale is in arcsec.

follow from these definitions, assuming a fixed excitation ratio and $\mathrm{CO}-$ to- $\mathrm{H}_{2}$ conversion ratio.

To gain insight on the possible $\mathrm{H}_{2}$ column densities of the torus, we computed the average $\mathrm{H}_{2}$ surface densities over the central beam on the highest $\mathrm{CO}(3-2)$ resolution maps (TM1). The resulting $\mathrm{H}_{2}$ colum densities are displayed in Table 4 together with the size of the beam in parsec. These column densities are lower limits on the actual molecular surface densities, both because of possible dilution in the beam, and also possible missing flux. Conversely, they might be sometimes an overestimation, if the $\mathrm{CO}(3-2)$ line becomes partially optically thin, due to high gas temperature and large turbulent line-width. In either case, from these central column densities, we can derive the molecular mass enclosed in the central beam, $M_{\text {cent }}$, displayed in Table 4. For NGC 1365, the central beam is empty, and the column density was taken from the peak surface density of the torus. The value of $M_{\text {cent }}$ is in general much lower than the expected central black hole mass, except in the case of NGC 613. This does not affect our BH mass estimation, since the gas mass is taken into account for the gravitational potential.

It is interesting to compare the $\mathrm{H}_{2}$ column densities inferred from our molecular measurements with the column densities derived from X-ray absorption. While our derived column density towards NGC 613 nucleus would suggest that it is Compton thick, Castangia et al. (2013) find NH values from X-ray spectral fitting that are lower, $\sim 4 \times 10^{23} \mathrm{~cm}^{-2}$. This may be explained by a clumpy torus. Nardini et al. (2015) examine NGC 1365 during a Compton-thick state, but NGC 1365 is a well-known "changinglook" AGN (Matt et al. 2003). In NGC 1566, Kawamuro et al. (2013) find a NH column of $\sim 3 \times 10^{22} \mathrm{~cm}^{-2}$ (with a $26 \%$ covering fraction). For NGC 1672, Jenkins et al. (2011) find inconclusive evidence for a Compton-thick X-ray source, although there is some evidence for $\mathrm{NH} \sim 2 \times 10^{24} \mathrm{~cm}^{-2}$ in agreement with our finding (de Naray et al. 2000). Jiménez-Bailón et al. (2005) find $\mathrm{NH} \sim 3 \times 10^{22} \mathrm{~cm}^{-2}$ for the hard nuclear source in NGC 1808. In summary, our very high-resolution data are not directly comparable to the X-ray measurements possible up to now. Our results are lower limits, allowing quite high column densities in some regions of the torus, but the latter can be quite clumpy. To be compatible with the X-ray estimations, the covering or surface filling factor has to be on the order of 0.01-0.1.

When there is a nuclear spiral structure, we have identified the torus as the nuclear component inside this structure (NGC 613, 1672, and 1808). It is likely that the torus is replenished in gas through this spiral structure. In that sense, the torus is the last axisymmetric structure before the nucleus. It is however difficult to define a typical torus in Seyfert galaxies: according to the larger scale dynamics (resonances, nuclear rings, or spirals), the size of the torus may vary widely. Other tori in the literature of even more varying sizes have been found: if the torus in NGC 1068 is comparable (4 pc radius, García-Burillo et al. 2016, or an extended one in the near-infrared of 27 pc radius Gratadour et al. 2015), others are larger, from $30-100 \mathrm{pc}$ in the $\mathrm{OH}$-megamasers of Mrk 231 (Klöckner et al. 2003), or smaller (1 pc for Circinus, Tristram et al. 2014, as well as Seyfert with $\mathrm{H}_{2} \mathrm{O}$ masers, with molecular transition at a fraction of a pc Madejski et al. 2000; Herrnstein et al. 2005). It is not yet clear whether the obscuring structure must be a thick doughnut-shaped torus, or could be a warped thin disk (e.g., Elitzur \& Shlosman 2006). In NGC 3079, the torus has been identified with clumps elevated from the warped and flared disk, at $0.5 \mathrm{pc}$, forming a geometrically thick structure of a few $10^{6} M_{\odot}$ (Kondratko et al. 2005).

\subsection{Determination of the mass of the black hole}

With the obtained ALMA spatial resolution, up to $2 \mathrm{pc}$ for NGC 1566, we are now reaching the sphere of influence (SoI) of the black holes in our nearby Seyfert galaxies. The SoI is defined as the region inside which the gravity of the black hole of mass $M_{\mathrm{BH}}$ dominates the gravitational potential of the host galaxy. There are two possible expressions for the SoI, one is from Merritt (2004), when the stellar mass inside the SoI1 $M_{*}(r<\mathrm{SoI} 1)$ is twice $M_{\mathrm{BH}}$, and the second involves the central velocity dispersion of the stars $\sigma_{v}$, i.e., $\mathrm{SoI} 2=G M_{\mathrm{BH}} / \sigma_{v}^{2}$. We display both in Table 6 . In the following, we also consider for the gravitational potential and the computation of the black hole mass; the example of NGC 1068 is presented here in comparison with the seven galaxies. Our previous high-resolution ALMA observations of its molecular torus have also reached the BH SoI (García-Burillo et al. 2016, and in prep.).

\subsubsection{Model of the gravitational potential}

To compute the stellar distribution of stars in each of our galaxies, we use the $\mathrm{S}^{4} \mathrm{G} 3.6 \mu \mathrm{m}$ infrared images and their galfit decomposition in bulge and disk components from Salo et al. (2015). More precisely, there are in most cases three components, including an additional bar component. These 
Table 4. Radii, masses, and inclinations of the molecular tori.

\begin{tabular}{|c|c|c|c|c|c|c|c|c|c|c|}
\hline Galaxy & $\begin{array}{l}\text { Radius } \\
\text { (pc) }\end{array}$ & $\begin{array}{l}\mathrm{S}(\mathrm{CO}) \mathrm{dV} \\
\mathrm{Jy} \mathrm{km} \mathrm{s}^{-1}\end{array}$ & $\begin{array}{l}\mathrm{M}\left(\mathrm{H}_{2}\right)^{a} \\
10^{7} \mathrm{M}_{\odot}\end{array}$ & $\begin{array}{l}\text { inc }\left(^{\circ}\right) \\
\text { torus }\end{array}$ & $\begin{array}{l}\mathrm{PA}\left({ }^{\circ}\right) \\
\text { torus }\end{array}$ & $\begin{array}{c}\text { inc }\left({ }^{\circ}\right)^{b} \\
\text { gal }\end{array}$ & $\begin{array}{c}\text { Beam } \\
(\mathrm{pc})\end{array}$ & $\begin{array}{c}\log \mathrm{NH}_{2} \\
\left(\mathrm{~cm}^{-2}\right)\end{array}$ & $\begin{array}{c}M_{\text {cent }} \\
10^{6} M_{\odot}\end{array}$ & $\begin{array}{l}\text { off-centering } \\
(\mathrm{pc})\end{array}$ \\
\hline NGC 613 & $14 \pm 3$ & $56 \pm 20$ & $3.9 \pm 1.4$ & $46 \pm 7$ & $0 \pm 8$ & 36 & 6.2 & $25.3 \pm .001$ & 10. & 42. \\
\hline NGC 1326 & $21 \pm 5$ & $18 \pm 2$ & $0.95 \pm 0.1$ & $60 \pm 5$ & $90 \pm 10$ & 53 & 5.3 & $23.9 \pm .02$ & 0.3 & 21. \\
\hline NGC 1365 & $26 \pm 3$ & $10 \pm 3$ & $0.74 \pm 0.2$ & $27 \pm 10$ & $70 \pm 10$ & 63 & 6.3 & $23.5 \pm .01$ & 0. & 86. \\
\hline NGC 1433 & - & - & - & - & - & 67 & 2.9 & $23.5 \pm 0.1$ & 0.04 & - \\
\hline NGC 1566 & $24 \pm 5$ & $72 \pm 10$ & $0.88 \pm 0.1$ & $12 \pm 12$ & $30 \pm 10$ & 48 & 1.7 & $24.5 \pm .01$ & 0.1 & 7. \\
\hline NGC 1672 & $27 \pm 7$ & $80 \pm 9$ & $2.5 \pm 0.3$ & $66 \pm 5$ & $0 \pm 10$ & 28 & 4.0 & $24.3 \pm .01$ & 0.4 & 27. \\
\hline NGC 1808 & $6 \pm 2$ & $46 \pm 6$ & $0.94 \pm 0.1$ & $64 \pm 7$ & $65 \pm 8$ & 84 & 3.1 & $24.6 \pm .004$ & 0.5 & 58. \\
\hline
\end{tabular}

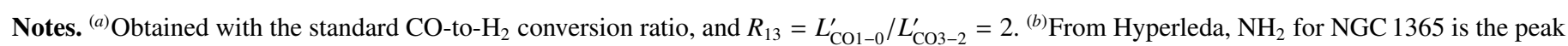
of the torus, but the central beam is empty. $\mathrm{NH}_{2}$ for $\mathrm{NGC} 1433$ is only that of the central arm. The errors on $\mathrm{NH}_{2}$ do not include the $\mathrm{CO}$-to- $\mathrm{H}_{2}$ conversion factor.

components are determined by their Sersic index, their magnitude, their effective radius, and the axis ratio. In two galaxies only, there is also a point-source component corresponding to $7 \%$ and $4 \%$ of the total mass respectively for NGC 1365 and NGC 1566. In these cases, we represented this component by an exponential, with effective radius equal to the PSF of the $3.6 \mu \mathrm{m}$ observations, which has a FWHM of 2.1" (Salo et al. 2015). All disks are exponential. As for bars, the adopted distribution is the modified Ferrers ellipsoids (Ferrers 1877), with a surface density varying as $\left[1-\left(\frac{r}{R_{\mathrm{bar}}}\right)^{2}\right]^{2}$, where $R_{\mathrm{bar}}$ is its outer truncation radius. We adopt a mass-to-light ratio of $M / L=0.5 M_{\odot} / L_{\odot}$ for this $3.6 \mu \mathrm{m}$ band (e.g., Sani et al. 2011; McGaugh \& Schombert 2014; Lelli et al. 2016). All the adopted mass components for our seven galaxies, with NGC 1068 added for comparison, are displayed in Table 5.

To have a first estimation of the black hole masses, it is interesting to exploit the well-known $M_{\mathrm{BH}}-\sigma$ relation for classical bulges (e.g., Tremaine et al. 2002; Marconi \& Hunt 2003; Gültekin et al. 2009). We use the hyperleda compilation (Makarov et al. 2014) for the central velocity dispersion, and also Garcia-Rissmann et al. (2005) when the data are not present in this data base. To derive the $\mathrm{BH}$ mass, we use the recent $M_{\mathrm{BH}}-\sigma$ relation,

$\log M_{\mathrm{BH}}\left(M_{\odot}\right)=8.5 \pm 0.05+(4.41 \pm 0.29) \log \left(\frac{\sigma_{v}}{200 \mathrm{~km} \mathrm{~s}^{-1}}\right)$,

compiled by Kormendy \& Ho (2013). The resulting masses are listed in Table 6, together with an indication of the bulge-to-black hole mass ratio (according to the $\mathrm{S}^{4} \mathrm{G} 3.6 \mu \mathrm{m}$ bulge decomposition) and the derived estimation of the SoI radius. The derived mass models from Table 5, including the BH masses of Table 6 are displayed in Fig. 8. The individual components from the galfit $3.6 \mu \mathrm{m}$ models are color-coded, and the vertical axis shows the SMBH versus galaxy mass ratios. From these values, it is clear that the ALMA observations can sample the dynamics inside the $\mathrm{BH}$ SoI in all galaxies. It can be seen in Fig. 8 that the enclosed stellar mass is not dominant in the regions sampled by the molecular tori, and the uncertainties on the determination of the stellar masses will not have a large influence on our modeling.

Many studies have shown that pseudo-bulges and/or barred galaxies may be offset from the main $M_{\mathrm{BH}^{-}} \sigma$ relation (Sani et al. 2011; Graham et al. 2011; Kormendy \& Ho 2013; Ho \& Kim 2014). Some of our galaxies may be in this category, and thus these first BH masses may be overestimated. It is therefore interesting to compare these values with several other BH mass estimations, such as the spiral pitch angle (Davis et al. 2014), or the
Table 5. Stellar mass components from $S^{4} \mathrm{G} 3.6 \mu \mathrm{m}$ (Salo et al. 2015).

\begin{tabular}{|c|c|c|c|c|c|}
\hline Galaxy & Comp. & $\begin{array}{c}R^{*} \\
(\mathrm{kpc}) \\
\end{array}$ & $\begin{array}{c}M_{*} \\
\left(10^{10} M_{\odot}\right)\end{array}$ & Sersic $n$ & $\begin{array}{c}\mathrm{B} / \mathrm{T} \\
M_{*}\end{array}$ \\
\hline \multirow{3}{*}{ N613 } & Bulge & 0.47 & 0.59 & 0.799 & 0.13 \\
\hline & Disk & 3.8 & 3.4 & 1.0 & 4.55 \\
\hline & Bar & 5.9 & 0.56 & & \\
\hline \multirow[t]{3}{*}{ N1326 } & Bulge & 0.43 & 0.63 & 1.167 & 0.32 \\
\hline & Disk & 2.7 & 0.99 & 1.0 & 1.98 \\
\hline & Bar & 4.1 & 0.36 & & \\
\hline \multirow{4}{*}{ N1365 } & Bulge & 1.1 & 2.3 & 0.857 & 0.25 \\
\hline & Disk & 8.2 & 5.0 & 1.0 & 8.66 \\
\hline & Bar & 7.8 & 1.3 & & \\
\hline & Nucleus & 0.25 & 0.06 & & \\
\hline \multirow[t]{3}{*}{ N1433 } & Bulge & 0.31 & 0.20 & 1.379 & 0.14 \\
\hline & Disk & 3.0 & 1.1 & 1.0 & 1.46 \\
\hline & Bar & 3.7 & 0.16 & & \\
\hline \multirow[t]{4}{*}{ N1566 } & Disk1 & 1.0 & 0.36 & 1.0 & 0.37 \\
\hline & Disk2 & 4.6 & 0.27 & 1.0 & 0.97 \\
\hline & Bar & 6.9 & 0.30 & & \\
\hline & Nucleus & 0.1 & 0.04 & & \\
\hline \multirow[t]{3}{*}{ N1672 } & Bulge & 0.36 & 0.54 & 0.749 & 0.23 \\
\hline & Disk & 3.3 & 1.5 & 1.0 & 2.32 \\
\hline & Bar & 4.0 & 0.28 & & \\
\hline \multirow[t]{3}{*}{ N1808 } & Bulge & 0.36 & 0.81 & 1.029 & 0.39 \\
\hline & Disk & 3.3 & 0.54 & 1.0 & 2.09 \\
\hline & Bar & 6.8 & 0.74 & & \\
\hline \multirow[t]{3}{*}{ N1068 } & Bulge & 0.58 & 2.4 & 1.181 & 0.38 \\
\hline & Disk1 & 0.94 & 2.3 & 1.0 & 6.30 \\
\hline & Disk2 & 7.0 & 1.6 & & \\
\hline
\end{tabular}

Notes. ${ }^{(*)}$ This is the effective radius $R_{\mathrm{e}}$ for a bulge, the scale-length $h_{\mathrm{r}}$ for an exponential disk, and the end radius $R_{\mathrm{bar}}$ for a bar. $M_{*}$ is the sum of all stellar components (bulge, disk, and bar) in $10^{10} M_{\odot}$.

Sersic index (Mutlu-Pakdil et al. 2016). These values are indicated in Table 7, and are indeed about 1-30 times (on average 4 times) below the previous ones.

From our ALMA data on the six galaxies with well-defined kinematic tori (the seven galaxies in Table 4, but without NGC 1433), we can try to derive the CO line kinematics inside the SoI. We use the position-velocity (PV) diagrams along the major axis, as employed by the WISDOM project (Onishi et al. 2017; Davis et al. 2017, 2018a) in deriving the supermassive black hole masses in three early-type galaxies from the molecular gas kinematics. We postpone to future work the modeling of the full 


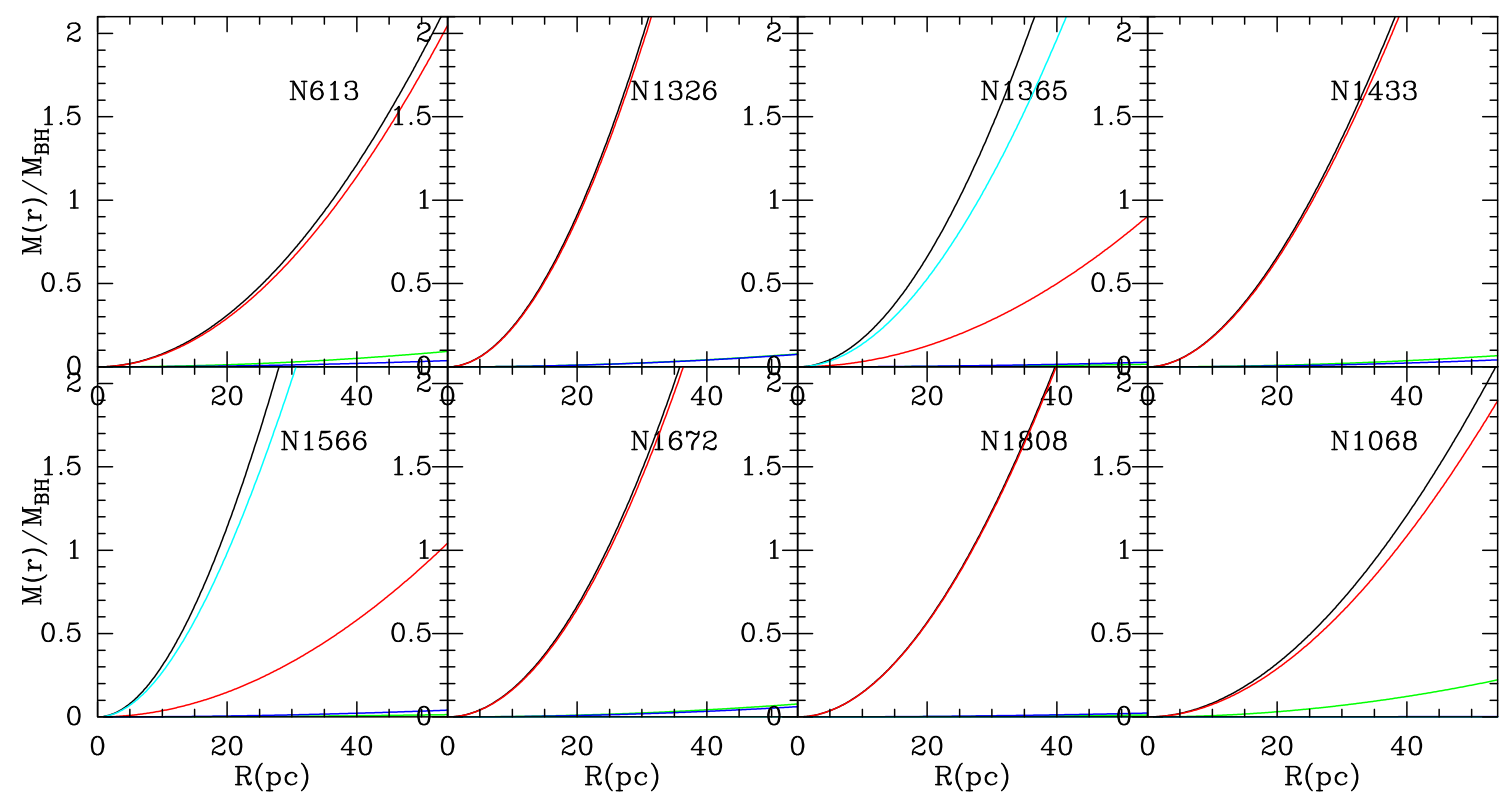

Fig. 8. Representation of the enclosed mass as a function of radius, taking into account every component of the galfit $3.6 \mu \mathrm{m}$ model (Table 5), with their respective geometry (spherical bulges, and flat disks and bars) for the eight galaxies. The vertical scale is normalized to their black hole mass, estimated from their central velocity dispersion (Table 6). The total mass is in black, the bulge in red, the disk in green, the bar in blue, and a possible nucleus in cyan.

Table 6. Central velocity dispersions and derived BH masses.

\begin{tabular}{lcccccc}
\hline \hline Galaxy & $\begin{array}{c}\sigma_{v} \\
\left(\mathrm{~km} \mathrm{~s}^{-1}\right) \\
(1)\end{array}$ & Ref. & $\begin{array}{c}M_{\mathrm{BH}} \\
\log M_{\odot} \\
(4)\end{array}$ & $\begin{array}{c}\mathrm{BH} / \mathrm{bul} \\
\%\end{array}$ & $\begin{array}{c}\text { SoI1 } \\
\mathrm{pc} \\
(5)\end{array}$ & $\begin{array}{c}\text { SoI2 } \\
\text { pc } \\
(6)\end{array}$ \\
\hline N613 & $122 \pm 18$ & $(1)$ & $7.57 \pm .27$ & 0.63 & 50 & 11. \\
N1326 & $111 \pm 14$ & $(1)$ & $7.40 \pm .22$ & 0.39 & 29 & 8.8 \\
N1365 & $141 \pm 19$ & $(1)$ & $7.84 \pm .26$ & 0.30 & 33 & 15. \\
N1433 & $113 \pm 3$ & $(2)$ & $7.40 \pm .03$ & 1.25 & 36 & 8.8 \\
N1566 & $98 \pm 7$ & $(1)$ & $7.13 \pm .10$ & 0.37 & 25 & 6.2 \\
N1672 & $111 \pm 3$ & $(2)$ & $7.40 \pm .03$ & 0.46 & 33 & 8.8 \\
N1808 & $138 \pm 9$ & $(1)$ & $7.79 \pm .13$ & 0.76 & 36 & 14. \\
N1068 & $174 \pm 9$ & $(1)$ & $8.23 \pm .13$ & 0.71 & 50 & 25. \\
\hline
\end{tabular}

Notes. The references for the central velocity dispersion in the second column are: (1) Hyperleda compilation (http://leda. univ-lyon1. $\mathrm{fr} /$ ). (2) Garcia-Rissmann et al. (2005) (cross-correlation method, CCM). The SoI is the radius of the sphere of influence of the black hole, the value in Col. 6 is from Merritt's definition, $M_{*}(r<$ SoI 1$)=2$ $M_{\mathrm{BH}}$, the value in Col. 7 is $\mathrm{SoI} 2=\mathrm{GM}_{\mathrm{BH}} / \sigma_{v}^{2}$.

velocity field of each galaxy because most objects are barred and subject to non-circular motions (S-shape), as seen in Figs. 2-5. As for the black-hole mass determination, we only focus on the central part of the PV diagram since the tori are frequently misaligned and tilted with respect to the main disk (Fischer et al. 2013). To determine the PA of the possibly decoupled tori, we plotted PV diagrams for a wide range of position angles (the first 36 separated by $10^{\circ}$, then with a separation of $5^{\circ}$ around the most probable PA), and selected the largest velocity gradient. Only the cubes with the most extended ALMA configuration were considered for this analysis (TM1). The analysis of the PV diagram in NGC 1808 did not allow the construction of an unambiguous mass model; the dynamics in the center is highly chaotic, probably due to the starburst and associated feedback. We therefore analyzed only the five remaining galaxies (the seven from Table 4, but without NGC 1433 and NGC 1808), with the methodology described here.
Table 7. BH masses from literature, and our derived ones.

\begin{tabular}{|c|c|c|c|c|c|}
\hline $\begin{array}{l}\text { Galaxy } \\
\text { (1) }\end{array}$ & $\begin{array}{c}\log M_{\mathrm{BH}} \\
M_{\odot} \\
(2)\end{array}$ & $\begin{array}{c}\log M_{\mathrm{BH}} \\
M_{\odot} \\
(3)\end{array}$ & $\begin{array}{l}\text { Ref. } \\
\text { (4) }\end{array}$ & $\begin{array}{c}\log M_{\mathrm{BH}} \\
M_{\odot} \\
(5)\end{array}$ & $\begin{array}{c}\log L_{\mathrm{AGN}} \\
\mathrm{erg} \mathrm{s}^{-1} \\
(6)\end{array}$ \\
\hline N 613 & $6.87 \pm .27$ & $7.60 \pm .35$ & (1) & $7.57 \pm .15$ & 42.1 \\
\hline N1326 & $7.47 \pm .17$ & $7.11 \pm .33$ & (2) & $6.81 \pm .2$ & 40.7 \\
\hline N1365 & $6.05 \pm .39$ & $6.30 \pm .4$ & (3) & $6.60 \pm .3$ & 42.8 \\
\hline N1433 & $6.61 \pm .37$ & $7.24 \pm .4$ & (4) & - & 40.0 \\
\hline N1566 & $7.11 \pm .32$ & $6.48 \pm .2$ & (5) & $6.83 \pm .3$ & 41.4 \\
\hline N1672 & $7.08 \pm .9$ & $6.00 \pm .6$ & (6) & $7.70 \pm .1$ & 39.3 \\
\hline N1808 & $6.74 \pm .35$ & $7.20 \pm .6$ & (7) & - & 40.6 \\
\hline N1068 & $6.93 \pm .37$ & $7.15 \pm .1$ & (8) & $7.17 \pm .2$ & 44.7 \\
\hline
\end{tabular}

Notes. Column 2 lists BH masses estimated from the spiral pitch angle (Davis et al. 2014), except for NGC 1326, from the Sersic index (Mutlu-Pakdil et al. 2016). Column 3 is from other estimations: (1) van den Bosch (2016), (2) Mould et al. (2012), (3) Risaliti et al. (2009), (4) Smajić et al. (2014), (5) Smajić et al. (2015), (6) Jenkins et al. (2011), (7) Busch et al. (2017), (8) Gallimore et al. (2001). Column 5 displays our best fit estimates from the molecular gas dynamics. For NGC 1068, the value comes from the $\mathrm{H}_{2} \mathrm{O}$ masers (e.g., Lodato \& Bertin 2003; Gallimore et al. 2016). Column 6 is the bolometric AGN luminosity (see Sect. 4.4.3).

\subsubsection{Fitting the PV diagrams, and the moment maps}

To be able to predict the gas dynamics in the inner nuclear disk with more flexibility, and with tilted decoupled dynamics, we performed numerical simulations of gas particles in a selected potential. We represent the gaseous disks by Miyamoto-Nagai disks of particles (Miyamoto \& Nagai 1975), with radial scales and heights corresponding to the observed tori. We used typically one million particles to ensure sufficient statistics. We plunge the gas disks into a potential made of the stellar distribution determined by galfit to each galaxy, as described in the previous section. The potential of the dark matter halo is negligible inside radii of $100 \mathrm{pc}$. We take into account the gas mass to build 


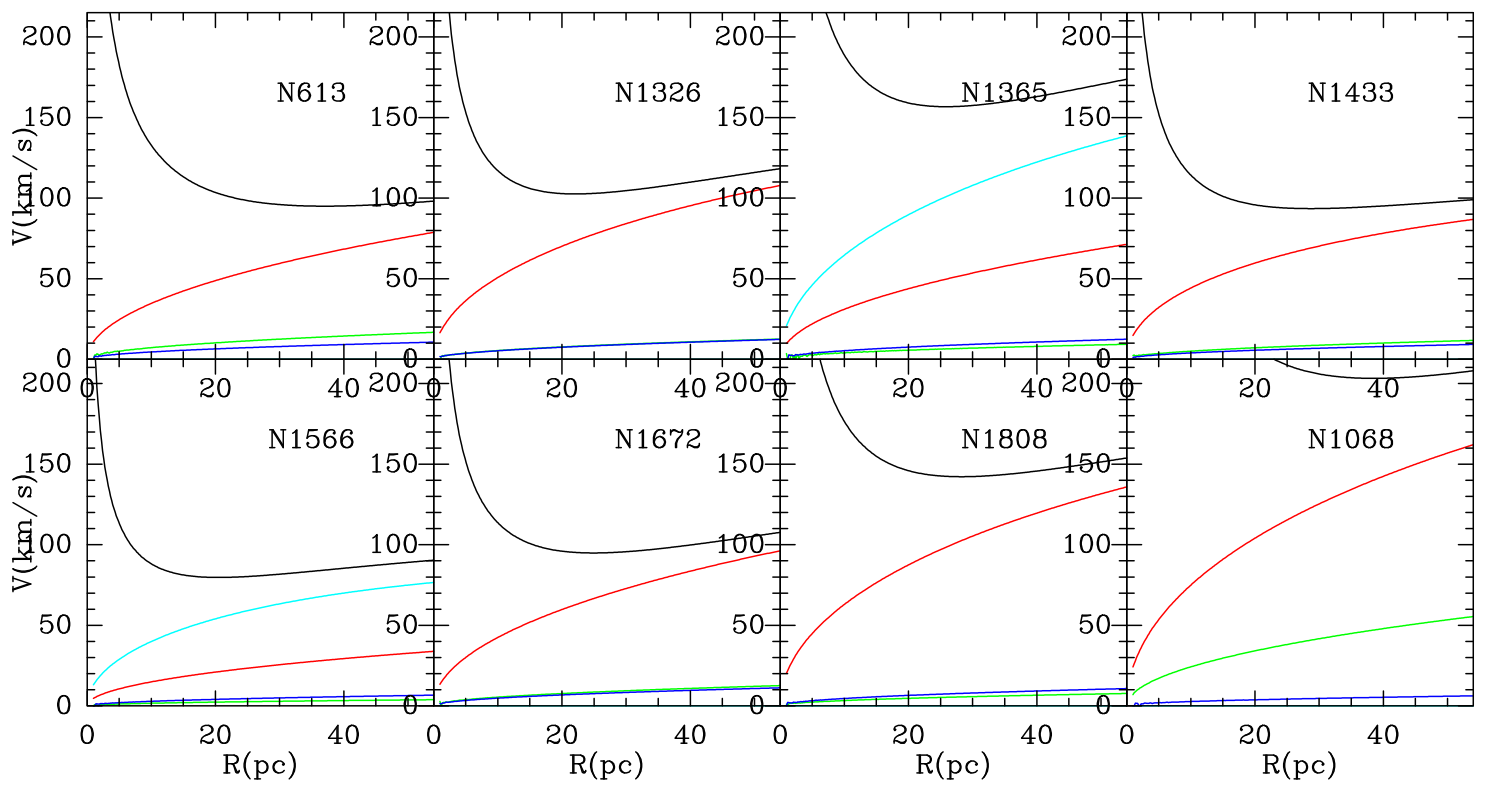

Fig. 9. Corresponding rotation curves for the eight galaxies, derived from the mass models in Fig. 8. The contribution of the three or four components is color-coded as in Fig. 8. The contribution of the black holes is included, with masses derived from the $M_{\mathrm{BH}}-\sigma$ relation (Table 6).

the total gravitational potential. The gas particles are distributed in circular orbits, in equilibrium with the total potential, with a velocity distribution corresponding to a Toomre Q-parameter of 1 . The ratio between tangential and radial velocity dispersion was taken from the epicyclic theory (e.g., Toomre 1964). It should be noted that we simulate only axisymmetric disks. Here we consider bar or spiral perturbations to be secondary, and will study their effects in future papers.

Figure 9 illustrates the small-scale rotation curves predicted by our mass models where the colors correspond to the same structural components as in Fig. 8. The quality of the potentials was checked by comparing at larger scales the modeled rotation curve with previous observations. These rotation curves come from $\mathrm{H} \alpha$, HI, or low-resolution $\mathrm{CO}$ observations, and do not have enough spatial resolution to sample the curve below $100 \mathrm{pc}$, but give a calibration at $0.2-1 \mathrm{kpc}$. Within these radii, the dark matter contribution is negligible. The curves are generally good approximations to those on larger scales, with some scatter which could come from the effect of non-circular motions. As will be seen below, the influence of the large-scale rotation has limited impact inside the sphere of influence of the black hole.

To compare the model to observations, we built data cubes by projecting the model on the sky, with the best fit large-scale inclinations and position angles (see above), and computing the lineof-sight velocity distribution. We select the same pixel size as the observed data cube (around 0.01 arsec according to each galaxy), and channels of $10 \mathrm{~km} \mathrm{~s}^{-1}$, and the data were smoothed to the observed beam (Table 1). The sizes in pixels of the cubes were between $(180,180,60)$ and $(360,360,60)$ to best sample the various tori studied. Because the gas distribution is not homogeneous, but asymmetric and patchy, and this impacts the mass-weighted velocity at each observed beam, we normalized the model cube to the zeroth moment map of the CO observations, pixel by pixel in this $2 \mathrm{D}$ projection. This plays the role of a multiplicative filter for our homogenous gas disks. This means that each $\mathrm{CO}$ spectrum at each position of the model is normalized to the observed integrated flux at this position.

We tested the methodology on the most regular PV diagram obtained, that of NGC 1672, as shown in Fig. 10. The three first moments of the model cube can be seen in Fig. 11. The first step is to run, as a comparison, a model with no black-hole, which is displayed in the left panel of Fig. 10. Then as a first estimate, the value derived from a standard $M_{\mathrm{BH}}-\sigma$ relation, as given in Table 6. We explore around this by small increments in $M_{\mathrm{BH}}$, which will allow us to obtain the best fit, maximizing the overlap between the contours of the model and the observed map in the PV diagram, and also fitting the three first moments. Typically a dozen values are explored for each parameter, which yield the best fit with error bars displayed in Table 7.

Two parameters were varied: the inclination of the central molecular disk/torus, and the mass of the black hole. To quantify the goodness of fit, we computed the least-squares values summing the difference of all pixels between the observed and model maps, either at 2D on the PV diagram, or the moment maps, or at 3D on the cubes. We concentrate on the nuclear part, with $3 \times 10^{4}$ pixels in $2 \mathrm{D}$ or $2 \times 10^{6}$ pixels in $3 \mathrm{D}$, corresponding to a region $120 \mathrm{pc}$ in diameter, with a resolution of $0.66 \mathrm{pc}$ and $10 \mathrm{~km} \mathrm{~s}^{-1}$. The model and observed maps are normalized to the same total flux over this region, and the squares difference is weighted by the observed flux in this pixel. The criterion is then to minimize the quantity: $\sum_{\text {pix }} w\left(F_{\text {obs }}-F_{\text {mod }}\right)^{2} / \sum_{\text {pix }} w F_{\text {obs }}^{2}$, with the weighting function $w=F_{\text {obs }}>0$. The result for NGC 1672 is illustrated in Fig. 12 for the PV diagram and the total cube.

The fit for the PV diagram and also for the 3D-cube tend to point towards large masses for the black hole. However, these high masses are not realistic, since they created a central velocity dispersion that is too large, as can be seen in Fig. 11. The best fit must therefore be a compromise between the PV diagram and the velocity dispersion map.

The same fitting procedure has been applied to NGC 613 (Figs. 13 and 14, showing in addition the perturbed velocity field and velocity dispersion due to the outflow), NGC 1326 (Figs. 15 and 16), NGC 1365, where the best compromise was obtained with the outer disk inclination (Figs. 17 and 18), and the same for NGC 1566 (Figs. 19 and 20). The morphology was too complex in NGC 1808 to obtain a satisfying fit, as mentioned in the previous section, perhaps because of supernovae feedback molecular flows there. 


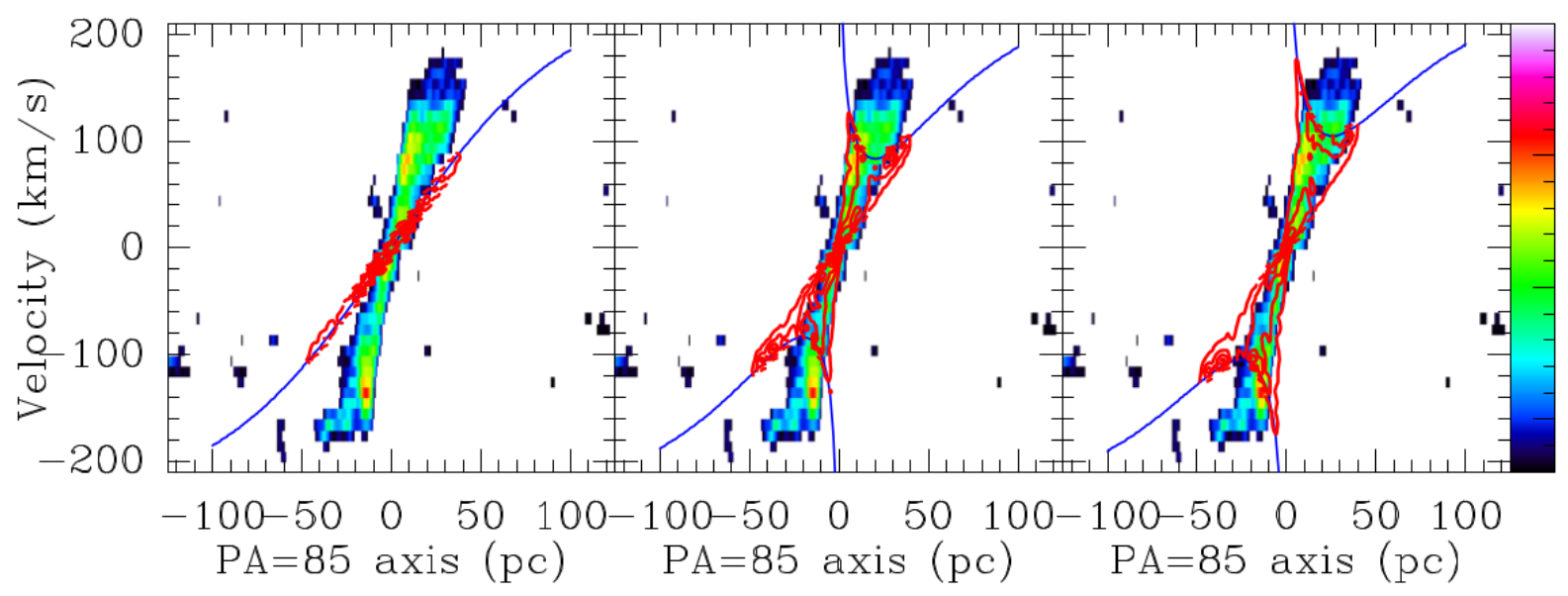

Fig. 10. Position-velocity diagram of the $\mathrm{CO}(3-2)$ line in NGC 1672, with a linear color scale (TM1 only). Superposed in red are the contours of the model without any black hole and torus inclination of $66^{\circ}$ (left panel), with a black hole as derived from the $M_{\mathrm{BH}}-\sigma$ relation (Table 6 ) of $2.5 \times 10^{7} M_{\odot}$ with $i=66^{\circ}$ (middle panel), and the best fit: a black hole of $5.0 \times 10^{7} M_{\odot}$, with $i=66^{\circ}$ (right panel). The mass model is that based on the galfit decomposition, and the predicted circular velocity is reproduced in blue lines (Fig. 9).

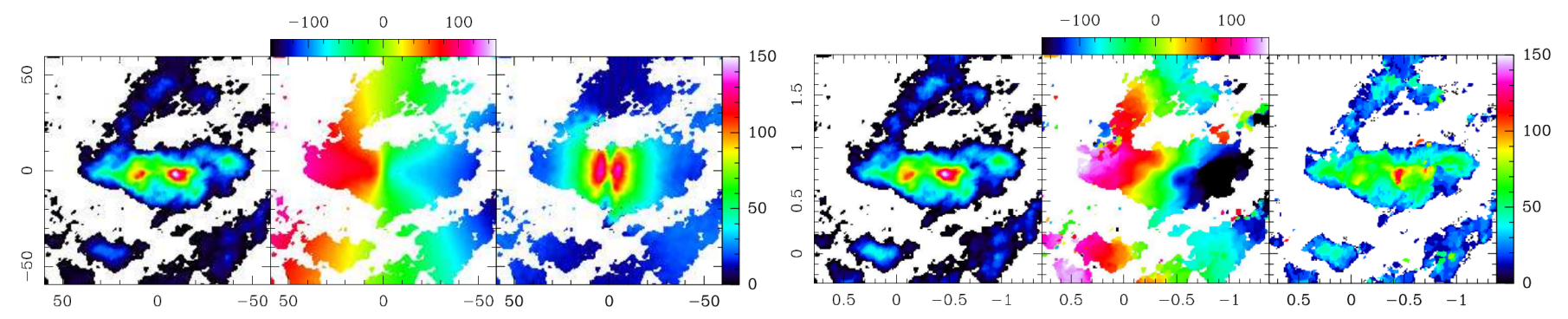

Fig. 11. Left panel: three first moments of the model cube for NGC 1672. The RA-Dec offsets are in parsec. The velocity color scales are in $\mathrm{km} \mathrm{s}^{-1}$. The cube corresponds to the best PV fit, with a BH mass of $5.0 \times 10^{7} M_{\odot}$, and an inclination of $66^{\circ}$. Right panel: three moments for the observations (TM1 only). The RA-Dec offsets are in arcsec with respect to the phase center.
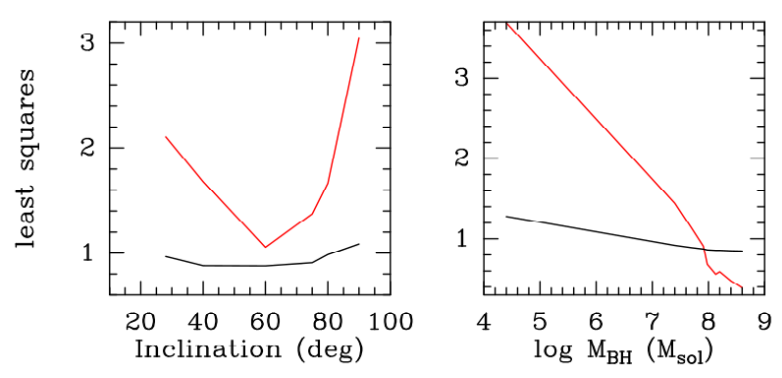

Fig. 12. Least-squares fitting between the observed and modeled PV diagram (black curve) and 3D cube (red curve) as a function of inclination of the torus (left panel) and the black hole mass (right panel). The criterion is defined in Sect. 4.4.2. The curves have been slightly translated vertically for clarity. If there is a minimum for inclination, there is none for $M_{\mathrm{BH}}$, and larger masses are preferred, as for NGC 1672 .

\subsubsection{Summary of results on black hole masses}

The black hole masses obtained are between $4 \times 10^{6}$ and $5 \times 10^{7} M_{\odot}$, in good agreement with the previous estimations of Tables 6 and 7: they have the tendency to follow the relation obtained for pseudo-bulge galaxies, determined by Ho \& Kim (2014). It is now fairly well established that classical bulges and ellipticals follow a tighter and shallower $M_{\mathrm{BH}}-\sigma$ relation than galaxies with pseudo-bulges (e.g., Graham et al. 2011, but see Bennert et al. 2015). The eight galaxies studied in this paper can all be considered pseudo-bulges: the Sersic index of their bulges are all close to 1 , and always $<1.4$ (Table 5). Also their bulge- to-disk ratios are all below 0.4 , which is the location of pseudobulges (e.g., Fisher \& Drory 2008; Gadotti 2009).

With the range of black hole masses found, the corresponding Eddington luminosities $L_{\text {Edd }}$ range from $1.3 \times 10^{11}$ and $1.6 \times 10^{12} L_{\odot}$, or $\log \left(L_{\mathrm{Edd}}\right)$ in $\mathrm{erg} \mathrm{s}^{-1}$ between 44.7 and 45.8 . From the X-ray luminosities listed in Table 1 and the bolometric corrections computed by Marconi et al. (2004), we estimated the AGN bolometric luminosities for all our galaxies, $L_{\mathrm{AGN}}$ (see Table 7 ). The Eddington ratio $L_{\mathrm{AGN}} / L_{\mathrm{Edd}}$ in our galaxies is therefore mostly $<<1$, namely between $\sim 0.2$ (NGC 1068) and $3 \times 10^{-7}$ (NGC 1672).

The positions of the galaxies studied here in the $M-\sigma$ diagram are displayed in Fig. 21, together with the compilation by van den Bosch (2016). The values measured recently with $\mathrm{CO}$ emission by the WISDOM collaboration are also plotted in blue: they apply to classical bulges with much higher black hole masses. Galaxies with pseudo-bulges and with lower masses are harder to determine, and their relation is affected by a larger scatter. In addition, barred galaxies appear in general below the standard $M_{\mathrm{BH}}-\sigma$ relation (e.g., Graham et al. 2011), which contributes to the scatter. The scatter in barred galaxies may be due to a varying velocity dispersion, because the orientation of the bar is random with respect to the line of nodes. In barred galaxies, gas driven into the center by gravity torques may boost a nuclear starburst, which produces a $\sigma$-drop (Wozniak et al. 2003). Also, in pseudo-bulge galaxies, the classical bulge is in general too light, and the black hole mass might be better compared to the total baryonic mass (Davis et al. 2018b). All 


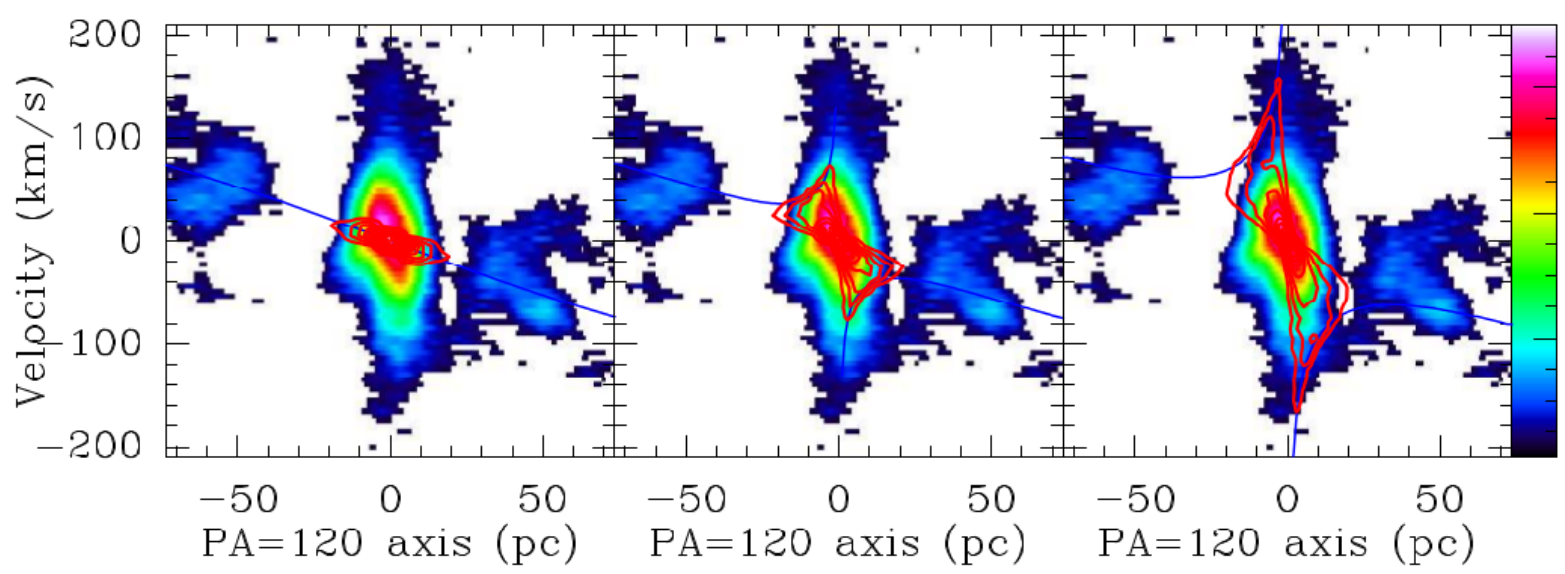

Fig. 13. Same as Fig. 10 for NGC 613, without any black hole (left panel), with a black hole of $7.4 \times 10^{6} M_{\odot}$ (middle panel), and the best fit: $3.7 \times 10^{7} M_{\odot}$, as derived from the $M_{\mathrm{BH}^{-}} \sigma$ relation (Table 6) (right panel). The inclination of the torus is $i=46^{\circ}$. The mass model is that based on the galfit decomposition, and the predicted circular velocity is reproduced in blue lines (Fig. 9).
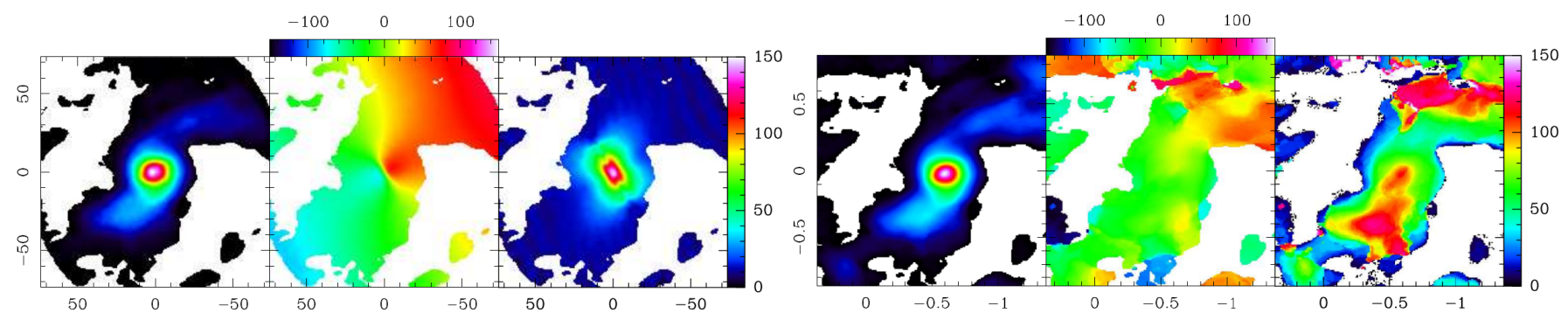

Fig. 14. Same as Fig. 11 for NGC 613 (left is the model, right the observations). The cube corresponds to the best PV fit, with a BH mass of $3.7 \times 10^{7} M_{\odot}$ and an inclination of $46^{\circ}$.

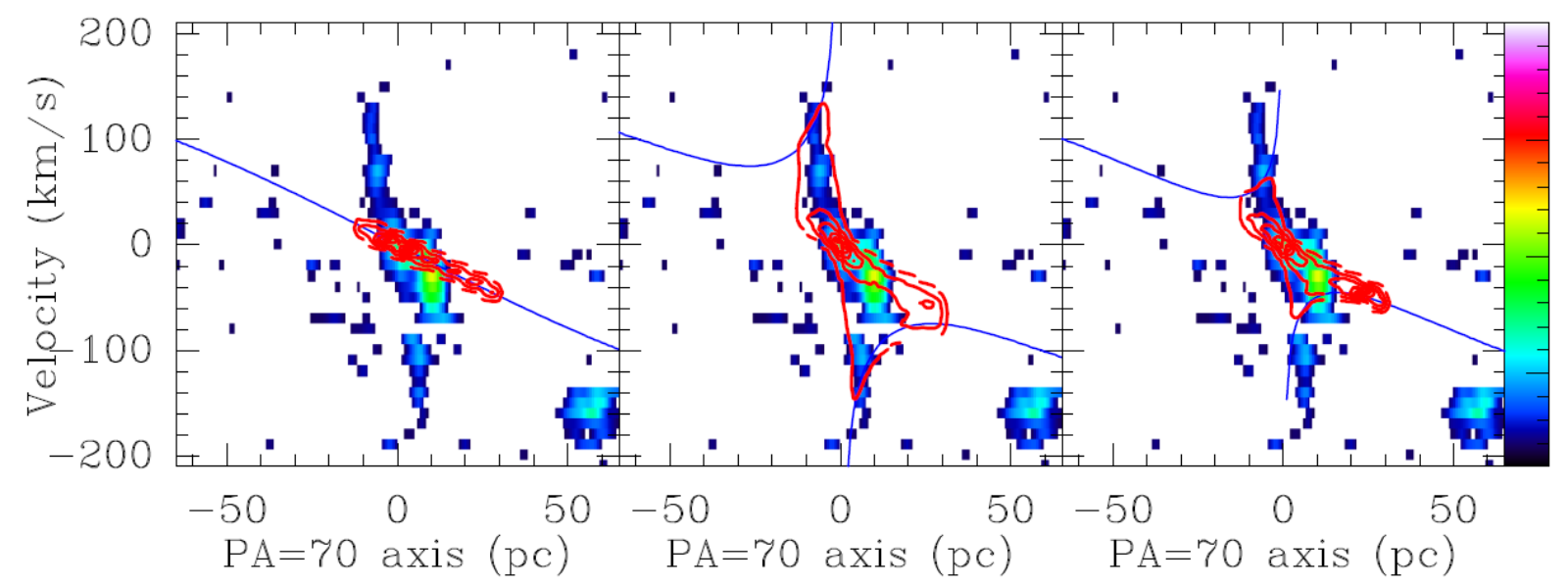

Fig. 15. Same as Fig. 10 for NGC 1326, without any black hole, $i=60^{\circ}$ (left panel); with a black hole of $3 \times 10^{7} M_{\odot}$ from the $M_{\mathrm{BH}}-\sigma$ relation, $i=60^{\circ}$ (middle panel); and the best fit: $6.5 \times 10^{6} M_{\odot}$, with $i=60^{\circ}$ (right panel).
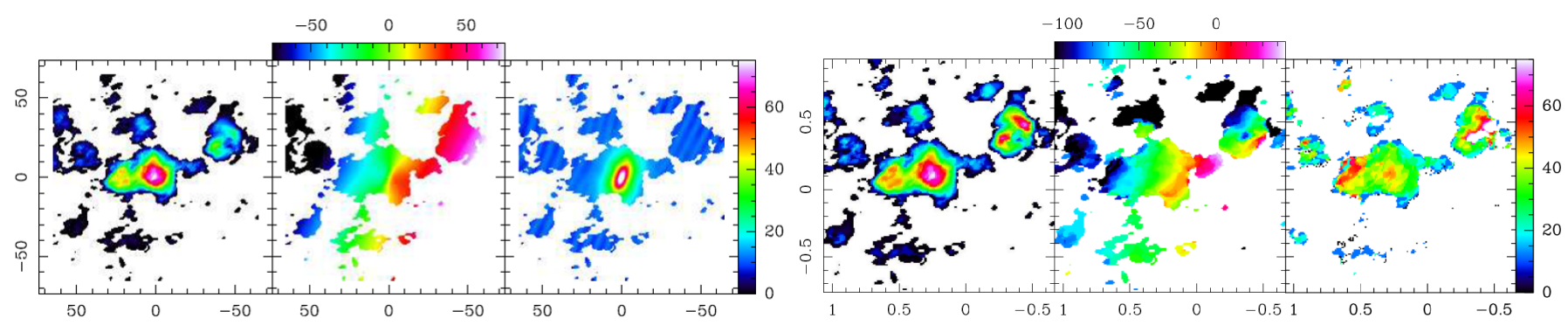

Fig. 16. Same as Fig. 11 for NGC 1326 (left is the model, right the observations). The cube corresponds to the best PV fit, with a BH mass of $6.5 \times 10^{6} M_{\odot}$ and an inclination of $60^{\circ}$. 


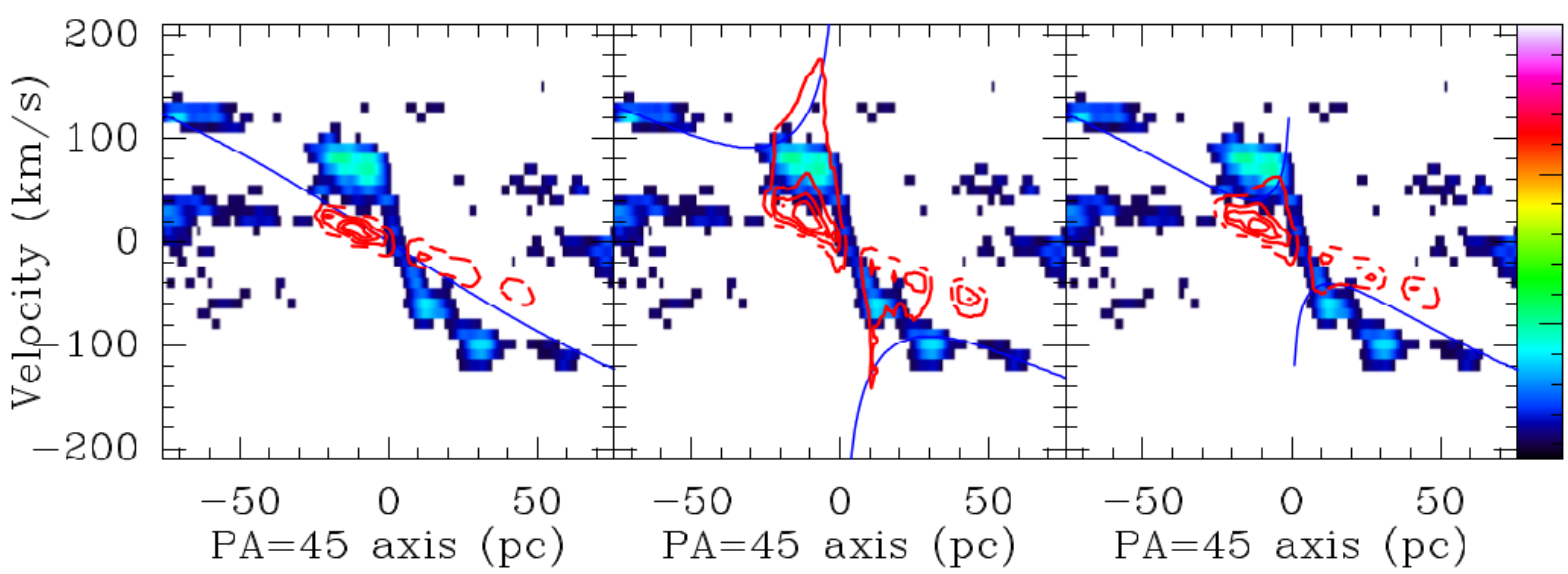

Fig. 17. Same as Fig. 10 for NGC 1365, without any black hole (left panel), with a black hole of $6.9 \times 10^{7} M_{\odot}$ from the $M_{\mathrm{BH}^{-}} \sigma$ relation $(m i d d l e$ panel), and the best fit: $4 \times 10^{6} M_{\odot}$ (right panel). The adopted inclination $i=63^{\circ}$ is from the galaxy to be conservative. Higher BH masss would be derived with the lower $27^{\circ}$ inclination estimated for the torus.
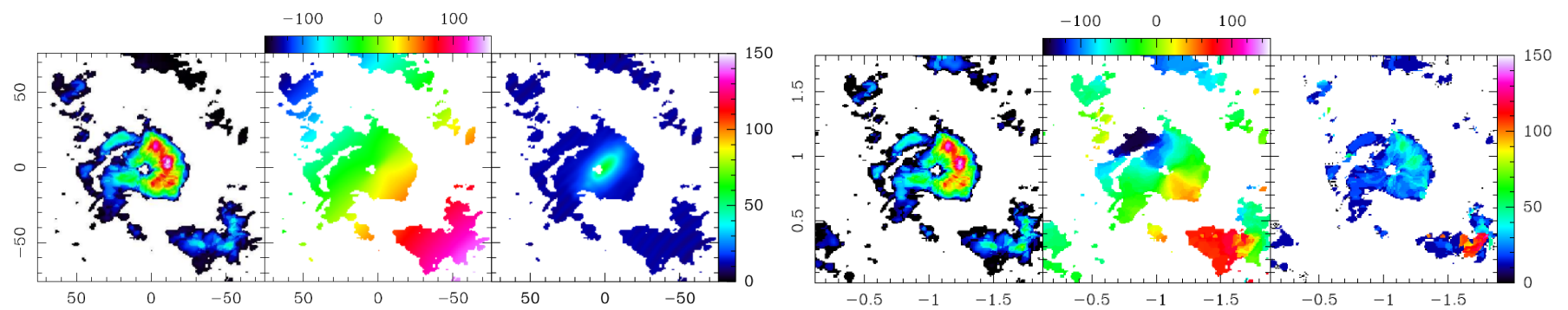

Fig. 18. Same as Fig. 11 for NGC 1365 (left is the model, right the observations). The cube corresponds to the best PV fit, with a BH mass of $4 \times 10^{6} M_{\odot}$ and an inclination of $63^{\circ}$.

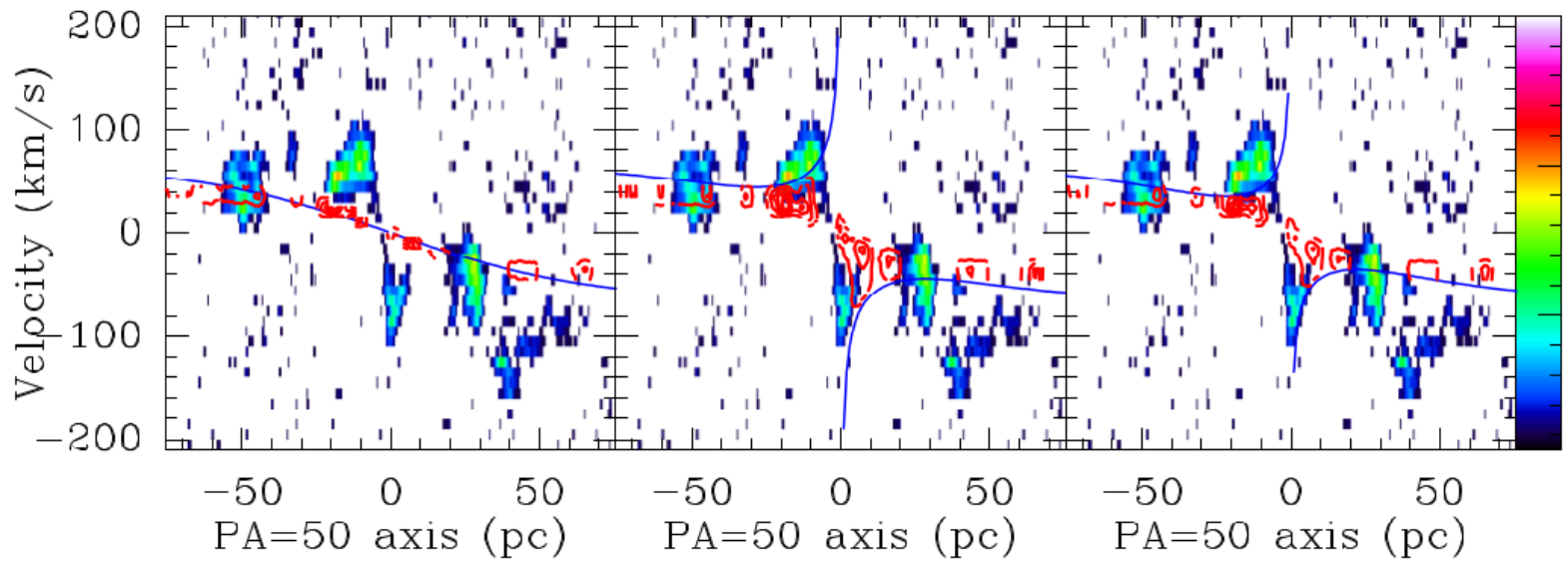

Fig. 19. Same as Fig. 10 for NGC 1566, without any black hole (left panel), with a black hole of $1.35 \times 10^{7} M_{\odot}$ from the $M_{\mathrm{BH}^{-}} \sigma$ relation (middle panel), and the best fit: $6.7 \times 10^{6} M_{\odot}$ (right panel). As for NGC 1365, the adopted inclination $i=48^{\circ}$ is from the galaxy.
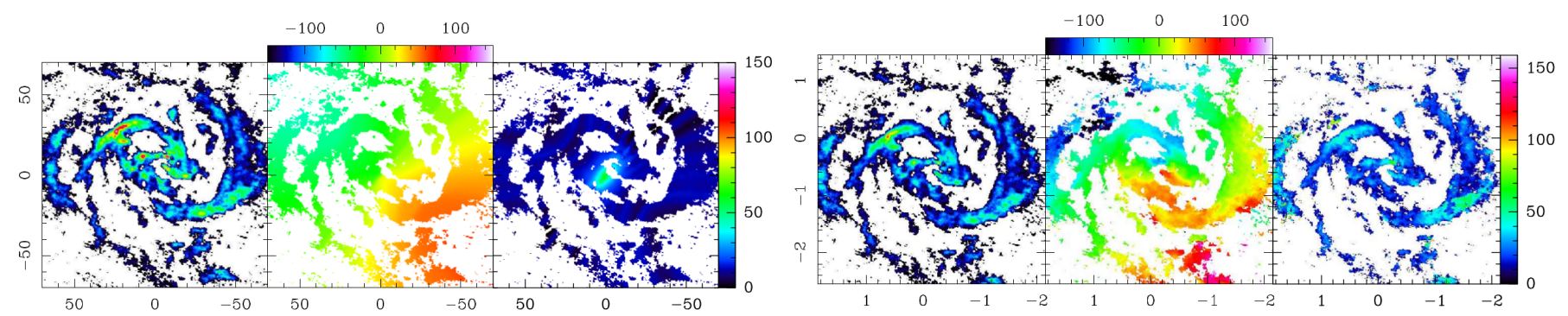

Fig. 20. Same as Fig. 11 for NGC 1566 (left is the model, right the observations). The cube corresponds to the best PV fit, with a BH mass of $6.7 \times 10^{6} M_{\odot}$ and an inclination of $48^{\circ}$. 


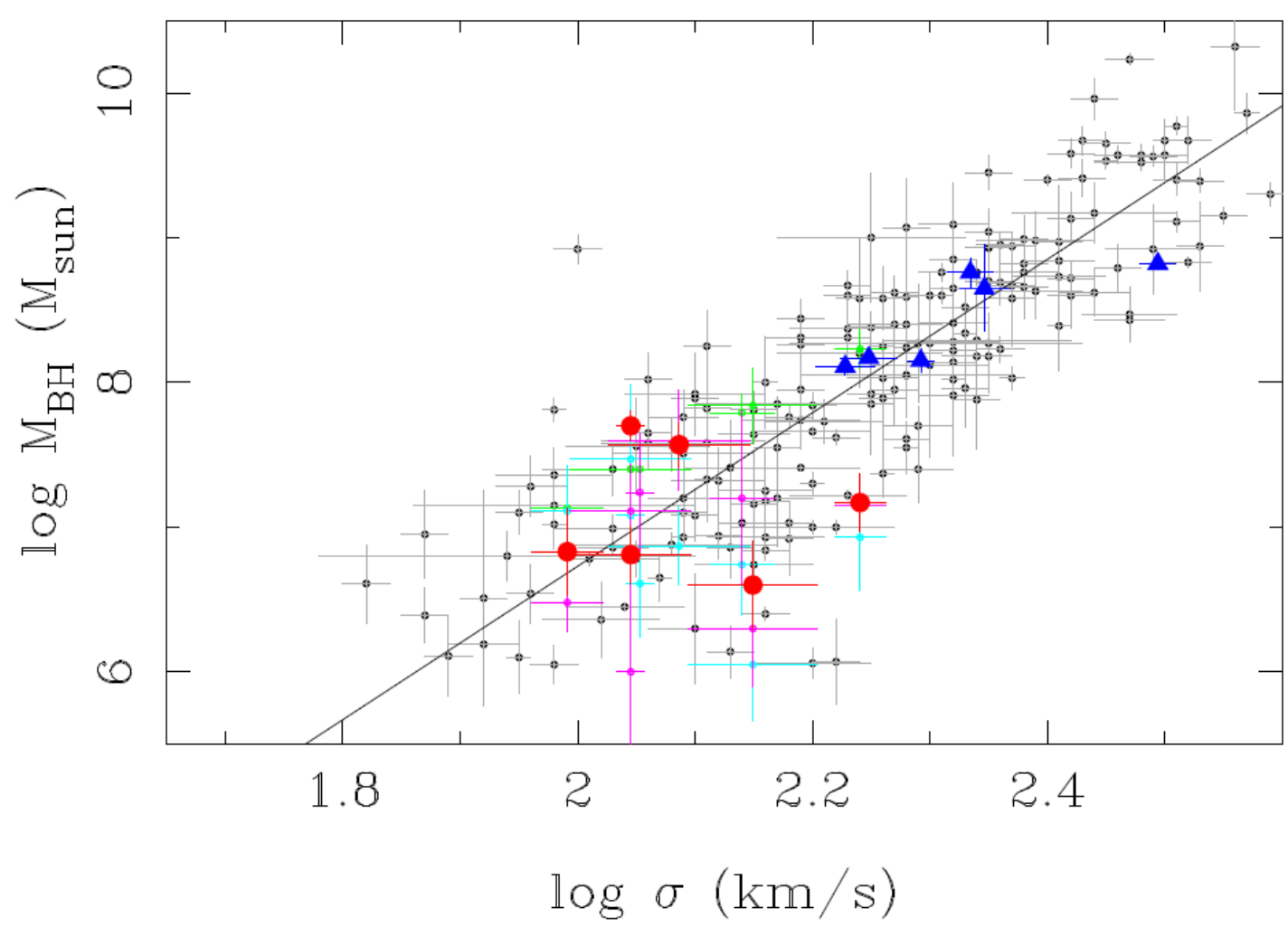

Fig. 21. Location of our galaxies in the $M_{\mathrm{BH}^{-}} \sigma$ relation, compiled by van den Bosch (2016), represented by the gray points and the fitted line, of slope 5.35. Our galaxies are in red, while in blue are plotted other values recently obtained from the CO line: NGC 1097 (Onishi et al. 2015), NGC 1332 (Barth et al. 2016), NGC 3665 (Onishi et al. 2017), NGC 4429 (Davis et al. 2018a), NGC 4526 (Davis et al. 2013 ), NGC 4697 (Davis et al. 2017). Previous estimations of the BH masses of our sample galaxies are also plotted in green (Table 6), turquoise and pink (Cols. 2 and 3 in Table 7).

eight galaxies studied in this paper are barred, and their scatter (Fig. 21) is not unexpected. It is, however, crucial to obtain $\mathrm{BH}$ masses in this low-mass end region to better understand the different processes for black hole growth, either through mergers (classical bulges) or secular evolution (pseudo-bulges).

It is interesting to compare the black hole masses derived in this paper with the previous estimations: in Fig. 21, the green points are the values derived from the $M_{\mathrm{BH}}-\sigma$ relation of Kormendy \& Ho (2013) and displayed in Table 6. These values are slightly above the $M_{\mathrm{BH}}-\sigma$ relation compiled by van den Bosch (2016). The turquoise points are the values from Col. 2 in Table 7 , estimated from the spiral pitch angle (Davis et al. 2014), and the pink points are the values from Col. 3 in Table 7 (various references). The red points appear less scattered.

\section{Discussion and summary}

We have presented our first ALMA results for a sample of 7 active galaxies, all low-luminosity AGN. The high spatial resolution allows to enter the sphere of influence of the central black holes, and to reveal the small-scale circumnuclear molecular structures that we associate with possible molecular tori. For 6 out of 7 galaxies, we indeed detect a nuclear disk, decoupled in morphology and dynamics from the main disk. Such a structure is also detected in an eighth galaxy studied by our group, NGC 1068 (García-Burillo et al. 2016, and in prep.).

\subsection{Characteristics of the tori}

The decoupled molecular tori (Table 4) may correspond to the required obscuration for type $2 \mathrm{AGN}$, even if they do not have the expected inclination for a type 2 classification. They sometimes show a gas hole or depletion in the center (NGC 1365, NGC 1566, NGC 1672) which make them approach a doughnut morphology. Their sizes extend over a wide range, from 6 to $27 \mathrm{pc}$, but their masses are less scattered from $\sim 1$ to $4 \times 10^{7} \mathrm{M}_{\odot}$. There is no relation to the total stellar masses of the galaxies, which vary from 1 to $9 \times 10^{10} M_{\odot}$ (Table 5). The mass of the gas in the molecular tori appears slightly anticorrelated to the AGN strength, as traced by the X-ray luminosity (Table 1), although with large scatter. More galaxies are needed to build a relation.

\subsubsection{Orientation of the tori}

One of the striking results emerging of these ALMA observations is that the gas in the molecular tori is rarely aligned with the main disk, but present different inclination and position angles. This decoupling was already observed for instance in NGC 1068 (Gratadour et al. 2015; García-Burillo et al. 2016). Given the widely different scales, $3-30 \mathrm{pc}$ for the nuclear disks and $1-10 \mathrm{kpc}$ for the main disk, the dynamical timescales are largely different, from $\sim 1$ to $300 \mathrm{Myr}$. It is then natural that these scales may decouple. It is sufficient that the central disk accretes some gas infalling with some angle to the plane for it to warp and change its orientation, averaging its old angular momentum vector with the tilted one from the newly accreted gas. The accretion could come either from the kpc scales or from stellar mass loss or feedback from its own star formation (e.g., Emsellem et al. 2015). Alternatively, the tori could be unstable to non-axisymmetric modes growing on a dynamical timescale (Papaloizou \& Pringle 1984). 


\subsubsection{Asymmetries: offsets and lopsidedness}

In addition, the molecular tori display asymmetries and offcentering. These circumnuclear disks are frequently located inside a star-forming ring, coincident with the ILR of the bar, but are not centered in the ring (for instance NGC 1326). The distance between the disk center (scale of a few hundred pc) and the AGN or torus center (which coincide) is displayed in Table 4. They are on the order of a few tens of pc, i.e., $\sim 10 \%$ of the scale of the ILR rings. This lopsidedness implies that the black hole is also wandering with a small amplitude around the center of mass of the galaxy. Several mechanisms for this offcentering and implied $\mathrm{BH}$ oscillations have been reviewed by Jog \& Combes (2009). The off-centering and wandering, characteristic of a Keplerian potential, may be explained by the eccentric instability proposed by Shu et al. (1990), when the masses of the central object and the disk are comparable. This mechanism occurs with the exchange of angular momentum between the central mass and the disk (Woodward et al. 1994). It will be interesting to establish whether the stellar component also displays $m=1$ instabilities, as the M 31 nucleus does, for example (e.g., Bender et al. 2005). These instabilities may help to fuel the AGN as predicted by Hopkins et al. (2012).

\subsubsection{Cases of the nuclear spirals}

In two cases, NGC 613 and NGC 1566, the CO emission has revealed a nuclear spiral, which has the distinction of being trailing, like the large-scale one. Inside the nuclear ring at the ILR of the bar, a leading spiral is usually expected to develop transiently and generate positive torques, which drive the inner gas onto the ring. However, when the gravitational impact of the black hole is significant, the spiral can then be trailing and the torques negative, to fuel the nucleus (e.g., Buta \& Combes 1996). The special case of NGC 613 will be studied in a forthcoming paper (Audibert et al., in prep.).

\subsection{Black hole masses}

The estimation of the black hole masses from the gas kinematics suffers from specific uncertainties, discussed in detail by previous authors (Barth et al. 2016; Onishi et al. 2015, 2017; Davis et al. 2017, 2018a). They discuss in particular the problem of edge-on systems, which do not have enough resolution on the minor axis or spatial resolution, which is barely equal to the radius of the SoI of the black hole. Our galaxies do not suffer from either of these problems: the spatial resolution of our ALMA observations is sufficient to probe the SoI, and therefore the impact of the stellar potential, and the uncertainties on the stellar mass-to-light ratio are less important here. However, our $\mathrm{BH}$ mass estimates still have the uncertainties related to the distance scale, and the trade-off needed to accommodate both the PV diagram fits and the observed velocity dispersion maps as discussed below.

All our galaxies are barred, at various strength levels, and certainly some non-circular motions are present, and must be included in the uncertainties. A typical S-shaped velocity pattern is seen in the NGC 1672 circumnuclear region (Fig. 11). We postpone to further work the simulation of the non-circular motions in these barred galaxies, including both small and large scale.

Another caveat might come from the observed $\mathrm{CO}$ velocity dispersion: we tried to take into account this projected velocity dispersion when comparing the moment 2 of the data cubes with the moments of our models. However, the molecular gas is patchy, and especially inside $50 \mathrm{pc}$ in radius it is likely that there is an insufficient number of molecular clouds to sample all the velocity gradients of the inner regions. In the model with one million particles, we sample almost continuously the velocity gradient, and this results in a large apparent dispersion along the line of sight towards the center in the case of a massive black hole. This apparent dispersion is only due to a beam-smearing of the velocity gradient along the line of sight. The intrinsic dispersion of the gas is negligible, between 2 and $10 \mathrm{~km} \mathrm{~s}^{-1}$. It is even more negligible when there is a massive central mass, since then the value of $\kappa$ the epicyclic frequency is significantly elevated, and the critical velocity to stabilize the disk in the Toomre sense is very low. In some cases, even when the velocity gradient is high and can only be obtained with a massive central component, the observed dispersion is surprisingly low. There are cases where the molecular gas is depleted or absent in the center, which is also a factor that supresses the large dispersion. This has to be taken into account in the minimizing criteria.

Perhaps the most important uncertainty in the black hole mass determination of these low-mass late-type objects is the decoupling of the molecular torus. On the one hand, these nuclear disks are dense enough to give kinematical information and subsist in the SoI, but their true inclination and position angle might be quite different from those of the main disks. It thus requires more 3D data to be able to disentangle both the decoupled morphology and the actual motions around the black hole.

In summary, ALMA at high resolution brings a wealth of new information on the decoupled molecular tori near the black holes. In the present paper, we described the properties of the decoupled molecular tori and estimated the mass of the central black holes, when possible, as displayed in Fig. 21. In future work, we will address the fueling efficiency through torque computation, and the feedback efficiency by estimating the gas outflows and their energetics in these low-luminosity AGN.

Acknowledgements. We warmly thank the referee for the constructive comments and suggestions. The ALMA staff in Chile and ARC-people at IRAM are gratefully acknowledged for their help in the data reduction. We particularly thank Philippe Salomé for his useful advice. SGB is thankful for the support from Spanish grant AYA2016-76682-C3-2-P. LKH acknowledges funding from the INAF PRIN-SKA 2017 program 1.05.01.88.04. This paper makes use of the following ALMA data: ADS/JAO.ALMA\#2015.0.00404.S, and ADS/JAO.ALMA\#2016.0.00296.S. ALMA is a partnership of ESO (representing its member states), NSF (USA), and NINS (Japan), together with NRC (Canada) and NSC and ASIAA (Taiwan), in cooperation with the Republic of Chile. The Joint ALMA Observatory is operated by ESO, AUI/NRAO, and NAOJ. The National Radio Astronomy Observatory is a facility of the National Science Foundation operated under cooperative agreement by Associated Universities, Inc. We used observations made with the NASA/ESA Hubble Space Telescope, and obtained from the Hubble Legacy Archive, which is a collaboration between the Space Telescope Science Institute (STScI/NASA), the Space Telescope European Coordinating Facility (ST-ECF/ESA), and the Canadian Astronomy Data Centre (CADC/NRC/CSA). We made use of the NASA/IPAC Extragalactic Database (NED) and of the HyperLeda database. This work was supported by the Programme National Cosmology et Galaxies (PNCG) of CNRS/INSU with INP and IN2P3, co- funded by CEA and CNES.

\section{References}

Aalto, S., Booth, R. S., Black, J. H., Koribalski, B., \& Wielebinski, R. 1994, A\&A, 286, 365

Aalto, S., Garcia-Burillo, S., Muller, S., et al. 2012, A\&A, 537, A44

Agüero, E. L., Díaz, R. J., \& Bajaja, E. 2004, A\&A, 414, 453

Alloin, D., Pelat, D., Phillips, M., \& Whittle, M. 1985, ApJ, 288, 205

Alonso-Herrero, A., Sánchez-Portal, M., Ramos Almeida, C., et al. 2012, MNRAS, 425, 311

Antonucci, R. R. J., \& Miller, J. S. 1985, ApJ, 297, 621 
Asmus, D., Gandhi, P., Smette, A., Hönig, S. F., \& Duschl, W. J. 2011, A\&A, 536, A36

Asmus, D., Hönig, S. F., \& Gandhi, P. 2016, ApJ, 822, 109

Bajaja, E., Wielebinski, R., Reuter, H.-P., Harnett, J. I., \& Hummel, E. 1995, A\&AS, 114, 147

Baribaud, T., Alloin, D., Glass, I., \& Pelat, D. 1992, A\&A, 256, 375

Barth, A. J., Boizelle, B. D., Darling, J., et al. 2016, ApJ, 822, L28

Bender, R., Kormendy, J., Bower, G., et al. 2005, ApJ, 631, 280

Bennert, V. N., Treu, T., Auger, M. W., et al. 2015, ApJ, 809, 20

Böker, T., Falcón-Barroso, J., Schinnerer, E., Knapen, J. H., \& Ryder, S. 2008, AJ, 135, 479

Braine, J., \& Combes, F. 1992, A\&A, 264, 433

Brandt, W. N., Halpern, J. P., \& Iwasawa, K. 1996, MNRAS, 281, 687

Busch, G., Eckart, A., Valencia-S, M., et al. 2017, A\&A, 598, A55

Buta, R., \& Combes, F. 1996, Fund. Cosmic Phys., 17, 95

Buta, R., Treuthardt, P. M., Byrd, G. G., \& Crocker, D. A. 2000, AJ, 120, 1289

Casey, C. M. 2012, MNRAS, 425, 3094

Castangia, P., Panessa, F., Henkel, C., Kadler, M., \& Tarchi, A. 2013, MNRAS, 436, 3388

Cicone, C., Maiolino, R., Sturm, E., et al. 2014, A\&A, 562, A21

Combes, F., García-Burillo, S., Casasola, V., et al. 2013, A\&A, 558, A124

Combes, F., García-Burillo, S., Casasola, V., et al. 2014, A\&A, 565, A97

Comerón, S. 2013, A\&A, 555, L4

Crocker, D. A., Baugus, P. D., \& Buta, R. 1996, ApJS, 105, 353

Dahlem, M., Aalto, S., Klein, U., et al. 1990, A\&A, 240, 237

Dahlem, M., Hartner, G. D., \& Junkes, N. 1994, ApJ, 432, 598

da Silva, P., Steiner, J. E., \& Menezes, R. B. 2017, MNRAS, 470, 3850

Davies, R. L., Groves, B., Kewley, L. J., et al. 2017, MNRAS, 470, 4974

Davis, T. A., Bureau, M., Cappellari, M., Sarzi, M., \& Blitz, L. 2013, Nature, 494, 328

Davis, B. L., Berrier, J. C., Johns, L., et al. 2014, ApJ, 789, 124

Davis, T. A., Bureau, M., Onishi, K., et al. 2017, MNRAS, 468, 4675

Davis, T. A., Bureau, M., Onishi, K., et al. 2018a, MNRAS, 473, 3818

Davis, B. L., Graham, A. W., \& Cameron, E. 2018b, ApJ, 869, 113

de Naray, P. J., Brandt, W. N., Halpern, J. P., \& Iwasawa, K. 2000, AJ, 119, 612

Díaz, R., Carranza, G., Dottori, H., \& Goldes, G. 1999, ApJ, 512, 623

Dumke, M., Nieten, C., Thuma, G., Wielebinski, R., \& Walsh, W. 2001, A\&A, 373,853

Elitzur, M., \& Shlosman, I. 2006, ApJ, 648, L101

Emsellem, E., Greusard, D., Combes, F., et al. 2001, A\&A, 368, 52

Emsellem, E., Renaud, F., Bournaud, F., et al. 2015, MNRAS, 446, 2468

Falcón-Barroso, J., Ramos Almeida, C., Böker, T., et al. 2014, MNRAS, 438, 329

Ferrers, N. M. 1877, Pure Appl. Math., 14, 1

Feruglio, C., Maiolino, R., Piconcelli, E., et al. 2010, A\&A, 518, L155

Fiore, F., Feruglio, C., Shankar, F., et al. 2017, A\&A, 601, A143

Fisher, D. B., \& Drory, N. 2008, AJ, 136, 773

Fischer, T. C., Crenshaw, D. M., Kraemer, S. B., \& Schmitt, H. R. 2013, ApJS, 209, 1

Gadotti, D. A. 2009, MNRAS, 393, 1531

Gallimore, J. F., Henkel, C., Baum, S. A., et al. 2001, ApJ, 556, 694

Gallimore, J. F., Elitzur, M., Maiolino, R., et al. 2016, ApJ, 829, L7

Garcia-Barreto, J. A., Dettmar, R.-J., Combes, F., Gerin, M., \& Koribalski, B. 1991, Rev. Mex. Astron. Astrofis., 22, 197

García-Burillo, S., Combes, F., Schinnerer, E., Boone, F., \& Hunt, L. K. 2005, A\&A, 441, 1011

García-Burillo, S., Combes, F., Usero, A., et al. 2014, A\&A, 567, A125

García-Burillo, S., Combes, F., Ramos Almeida, C., et al. 2016, ApJ, 823, L12

Garcia-Rissmann, A., Vega, L. R., Asari, N. V., et al. 2005, MNRAS, 359, 765

Graham, A. W., Onken, C. A., Athanassoula, E., \& Combes, F. 2011, MNRAS, 412, 2211

Gratadour, D., Rouan, D., Grosset, L., Boccaletti, A., \& Clénet, Y. 2015, A\&A, 581, L8

Greenhill, L. J., Gwinn, C. R., Antonucci, R., \& Barvainis, R. 1996, ApJ, 472, L21

Guilloteau, S., \& Lucas, R. 2000, ASP Conf. Ser., 217, 299

Gültekin, K., Richstone, D. O., Gebhardt, K., et al. 2009, ApJ, 698, 198

Hatziminaoglou, E., Fritz, J., \& Jarrett, T. H. 2009, MNRAS, 399, 1206

Herrnstein, J. R., Moran, J. M., Greenhill, L. J., \& Trotter, A. S. 2005, ApJ, 629, 719

Hjelm, M., \& Lindblad, P. O. 1996, A\&A, 305, 727

Ho, L. C., \& Kim, M. 2014, ApJ, 789, 17

Hopkins, P. F., Hayward, C. C., Narayanan, D., \& Hernquist, L. 2012, MNRAS, 420, 320

Hummel, E., \& Jorsater, S. 1992, A\&A, 261, 85

Imanishi, M., \& Wada, K. 2004, ApJ, 617, 214

Imanishi, M., Nakanishi, K., \& Izumi, T. 2016, ApJ, 822, L10

Imanishi, M., Nakanishi, K., Izumi, T., \& Wada, K. 2018, ApJ, 853, L25
Jenkins, L. P., Brandt, W. N., Colbert, E. J. M., et al. 2011, ApJ, 734, 33

Jiménez-Bailón, E., Santos-Lleó, M., Dahlem, M., et al. 2005, A\&A, 442, 861

Jog, C. J., \& Combes, F. 2009, Phys. Rep., 471, 75

Jungwiert, B., Combes, F., \& Axon, D. J. 1997, A\&AS, 125, 479

Kawamuro, T., Ueda, Y., Tazaki, F., \& Terashima, Y. 2013, ApJ, 770, 157

Kewley, L. J., Heisler, C. A., Dopita, M. A., et al. 2000, ApJ, 530, 704

Klöckner, H.-R., Baan, W. A., \& Garrett, M. A. 2003, Nature, 421, 821

Kondratko, P. T., Greenhill, L. J., \& Moran, J. M. 2005, ApJ, 618, 618

Kondratko, P. T., Greenhill, L. J., Moran, J. M., et al. 2006, ApJ, 638, 100

Koribalski, B., Dahlem, M., Mebold, U., \& Brinks, E. 1993, A\&A, 268, 14

Koribalski, B., Dettmar, R.-J., Mebold, U., \& Wielebinski, R. 1996, A\&A, 315, 71

Kormendy, J., \& Ho, L. C. 2013, ARA\&A, 51, 511

LaMassa, S. M., Cales, S., Moran, E. C., et al. 2015, ApJ, 800, 144

Lelli, F., McGaugh, S. S., \& Schombert, J. M. 2016, AJ, 152, 157

Lena, D., Robinson, A., Storchi-Bergmann, T., et al. 2016, MNRAS, 459, 4485

Lindblad, P. O. 1999, A\&ARv, 9, 221

Lodato, G., \& Bertin, G. 2003, A\&A, 398, 517

Mac Low, M.-M., \& McCray, R. 1988, ApJ, 324, 776

Madejski, G., Życki, P., Done, C., et al. 2000, ApJ, 535, L87

Makarov, D., Prugniel, P., Terekhova, N., Courtois, H., \& Vauglin, I. 2014, A\&A, 570, A13

Marconi, A., \& Hunt, L. K. 2003, ApJ, 589, L21

Marconi, A., Risaliti, G., Gilli, R., et al. 2004, MNRAS, 351, 169

Matt, G., Guainazzi, M., \& Maiolino, R. 2003, MNRAS, 342, 422

McElroy, R. E., Husemann, B., Croom, S. M., et al. 2016, A\&A, 593, L8

McGaugh, S. S., \& Schombert, J. M. 2014, AJ, 148, 77

McMullin, J. P., Waters, B., Schiebel, D., Young, W., \& Golap, K. 2007, ASP Conf. Ser., 376, 127

Merritt, D. 2004, Coevol. Black Holes Galaxies, 263

Miyamoto, M., \& Nagai, R. 1975, PASJ, 27, 533

Miyamoto, Y., Nakai, N., Seta, M., et al. 2017, PASJ, 69, 83

Mould, J., Reynolds, T., Readhead, T., et al. 2012, ApJS, 203, 14

Mutlu-Pakdil, B., Seigar, M. S., \& Davis, B. L. 2016, ApJ, 830, 117

Nardini, E., Gofford, J., Reeves, J. N., et al. 2015, MNRAS, 453, 2558

Onishi, K., Iguchi, S., Sheth, K., \& Kohno, K. 2015, ApJ, 806, 39

Onishi, K., Iguchi, S., Davis, T. A., et al. 2017, MNRAS, 468, 4663

Papadopoulos, P. P., van der Werf, P. P., Xilouris, E. M., et al. 2012, MNRAS, 426, 2601

Papaloizou, J. C. B., \& Pringle, J. E. 1984, MNRAS, 208, 721

Prieto, M. A., Reunanen, J., Tristram, K. R. W., et al. 2010, MNRAS, 402, 724

Reunanen, J., Kotilainen, J. K., \& Prieto, M. A. 2002, MNRAS, 331, 154

Risaliti, G., Miniutti, G., Elvis, M., et al. 2009, ApJ, 696, 160

Sakamoto, K., Ho, P. T. P., Mao, R.-Q., Matsushita, S., \& Peck, A. B. 2007, ApJ, 654,782

Salak, D., Nakai, N., Hatakeyama, T., \& Miyamoto, Y. 2016, ApJ, 823, 68

Salak, D., Tomiyasu, Y., Nakai, N., et al. 2017, ApJ, 849, 90

Salo, H., Laurikainen, E., Laine, J., et al. 2015, ApJS, 219, 4

Sánchez-Blázquez, P., Ocvirk, P., Gibson, B. K., Pérez, I., \& Peletier, R. F. 2011, MNRAS, 415, 709

Sanders, D. B., Mazzarella, J. M., Kim, D.-C., Surace, J. A., \& Soifer, B. T. 2003, AJ, 126, 1607

Sandqvist, A. 1999, A\&A, 343, 367

Sani, E., Marconi, A., Hunt, L. K., \& Risaliti, G. 2011, MNRAS, 413, 1479

Shu, F. H., Tremaine, S., Adams, F. C., \& Ruden, S. P. 1990, ApJ, 358, 495

Smajić, S., Moser, L., Eckart, A., et al. 2014, A\&A, 567, A119

Smajić, S., Moser, L., Eckart, A., et al. 2015, A\&A, 583, A104

Solomon, P. M., \& Vanden Bout, P. A. 2005, ARA\&A, 43, 677

Steer, I., Madore, B. F., Mazzarella, J. M., et al. 2017, AJ, 153, 37

Stevens, I. R., Forbes, D. A., \& Norris, R. P. 1999, MNRAS, 306, 479

Storchi-Bergmann, T., Rodriguez-Ardila, A., Schmitt, H. R., Wilson, A. S., \& Baldwin, J. A. 1996, ApJ, 472, 83

Surcis, G., Tarchi, A., Henkel, C., et al. 2009, A\&A, 502, 529

Tabatabaei, F. S., Weiß, A., Combes, F., et al. 2013, A\&A, 555, A128

Tacconi, L. J., Neri, R., Genzel, R., et al. 2013, ApJ, 768, 74

Tombesi, F., Cappi, M., Reeves, J. N., et al. 2010, A\&A, 521, A57

Toomre, A. 1964, ApJ, 139, 1217

Tremaine, S., Gebhardt, K., Bender, R., et al. 2002, ApJ, 574, 740

Tristram, K. R. W., Burtscher, L., Jaffe, W., et al. 2014, A\&A, 563, A82

Urry, C. M., \& Padovani, P. 1995, PASP, 107, 803

van den Bosch, R. C. E. 2016, ApJ, 831, 134

Veilleux, S., Cecil, G., \& Bland-Hawthorn, J. 2005, ARA\&A, 43, 769

Venturi, G., Marconi, A., Mingozzi, M., et al. 2017, Front. Astron. Space Sci., 4, 46

Woodward, J. W., Tohline, J. E., \& Hachisu, I. 1994, ApJ, 420, 247

Wozniak, H., Combes, F., Emsellem, E., \& Friedli, D. 2003, A\&A, 409, 469

Zhang, J. S., Henkel, C., Guo, Q., \& Wang, J. 2012, A\&A, 538, A152 